\title{
REVIEWS
}

Check for updates

\section{Multifunctional biomolecule nanostructures for cancer therapy}

Jing Wang $\mathbb{1}^{1,2,5}$, Yiye Li $\mathbb{1}^{1,2,5}$ and Guangjun Nie (1) ${ }^{1,2,3,4 凶}$

Abstract | Biomolecule-based nanostructures are inherently multifunctional and harbour diverse biological activities, which can be explored for cancer nanomedicine. The supramolecular properties of biomolecules can be precisely programmed for the design of smart drug delivery vehicles, enabling efficient transport in vivo, targeted drug delivery and combinatorial therapy within a single design. In this Review, we discuss biomolecule-based nanostructures, including polysaccharides, nucleic acids, peptides and proteins, and highlight their enormous design space for multifunctional nanomedicines. We identify key challenges in cancer nanomedicine that can be addressed by biomolecule-based nanostructures and survey the distinct biological activities, programmability and in vivo behaviour of biomolecule-based nanostructures. Finally, we discuss challenges in the rational design, characterization and fabrication of biomolecule-based nanostructures, and identify obstacles that need to be overcome to enable clinical translation.

${ }^{1}$ CAS Key Laboratory for Biomedical Effects of Nanomaterials \& Nanosafety, CAS Center for Excellence in Nanoscience, National Center for Nanoscience and Technology, China, Beijing, China.

${ }^{2}$ Center of Materials Science and Optoelectronics Engineering, University of Chinese Academy of Sciences, Beijing, China.

${ }^{3} \mathrm{GBA}$ Research Innovation Institute for Nanotechnology. Guangdong, China

${ }^{4}$ Henan Institute of Advanced Technology, Zhengzhou University, Zhengzhou, China.

${ }^{5}$ These authors contributed equally: Jing Wang, Yiye Li.

凶e-mail:niegj@nanoctr.cn

https://doi.org/10.1038/ s41578-021-00315-x
A major advantage of nanostructures in biomedical applications is their multifunctional capacity. The flexibility of nanomaterial design and modification allows us to exploit different nano-bio interactions with a single system. Such multifunctionality is particularly important for the treatment of complex and heterogeneous diseases, such as cancer. Numerous anticancer functionalities can be combined in a nanostructure-based therapy approach, including targeted delivery, sequential targeting, stimuli-responsiveness, theranostics, combination treatment and built-in logic gates, to realize refined therapeutic actions ${ }^{1-4}$. However, the application of nanostructures in cancer therapy also faces several challenges; for example, a series of biological barriers interfere with the transport of nanostructures to target tissues, limiting delivery efficiency ${ }^{5,6}$. In addition, nanoformulations are subject to reticuloendothelial clearance and often show limited tumour penetration, as compared with free drugs. Therefore, effort has been dedicated to the development of functionalities that specifically overcome or circumvent biological barriers, clearance and limited penetration into tissues. The great variety of nanomaterials further leads to diverse and complicated in vivo behaviours, and the introduction of multiple components can increase the risk of unpredicted loss of function or adverse effects. It is therefore important to apply multifunctional nanomaterial design judiciously, taking into consideration the particular properties of the nanomaterial or functional units and the specific medical requirements.

In cancer nanomedicine, natural and engineered biomolecules (that is, polysaccharides, nucleic acids, peptides and proteins) have been explored for nanostructure construction ${ }^{7-11}$. These biomolecules are generally biocompatible and biodegradable, and often intrinsically bioactive as targeting and/or therapeutic agents. The protein-based nanoformulation Abraxane (albumin-bound paclitaxel) is already used in the clinic ${ }^{12}$, and several biomolecule-derived nanostructures are currently in clinical trials (TABLE 1). Although liposomes and polymeric particles remain the nanoplatforms with the most translational success thus far $^{4,13}$, biomolecule-based building blocks offer specific properties that cannot be facilely reproduced in these synthetic materials. For example, the supramolecular interactions of peptide and nucleic acid materials can be precisely manipulated, and natural-protein-based nanosystems can mimic proteins in terms of molecular recognition or enzymatic activities. Importantly, multifunctional strategies can be designed using biomolecule-derived elements, each with distinct characteristics and limitations. However, a holistic view of biomolecule-based nanostructures in the broad context of cancer nanomedicine remains elusive. In this Review, we discuss biomolecule-based nanomaterials, highlighting specific multifunctional features in the context of cancer targeting and therapy.

\section{Challenges in cancer nanomedicine}

The fate of a nanosystem administered to an organism is influenced by a large network of factors. In particular, the efficiency of antitumour nanostructures is limited by difficulties in targeting, dynamic in vivo changes of the materials and multiple biological barriers. These complex and interconnected obstacles require multilayered 
Table 1 | Biomolecule-based nanostructures approved or in clinical trials for cancer therapy

\begin{tabular}{|c|c|c|c|c|c|c|c|}
\hline Biomolecule & Nanostructure & Name & $\begin{array}{l}\text { Therapeutic } \\
\text { modality }\end{array}$ & $\begin{array}{l}\text { Active } \\
\text { pharmaceutical } \\
\text { ingredient(s) }\end{array}$ & Indication(s) & Status & $\begin{array}{l}\text { ClinicalTrials } \\
\text { gov identifier }\end{array}$ \\
\hline Polysaccharide & $\begin{array}{l}\text { Cyclodextrin- } \\
\text { PEG copolymer } \\
\text { nanoparticle }\end{array}$ & $\begin{array}{l}\text { NLG207, } \\
\text { formerly } \\
\text { CRLX101 }\end{array}$ & $\begin{array}{l}\text { Targeted } \\
\text { molecular } \\
\text { therapy }\end{array}$ & $\begin{array}{l}\text { Camptothecin } \\
\text { (topoisomerase I } \\
\text { inhibitor) }\end{array}$ & Rectal cancer & Phase I/II & NCT02010567 \\
\hline \multirow[t]{6}{*}{ Protein } & \multirow[t]{6}{*}{$\begin{array}{l}\text { Albumin } \\
\text { nanoparticle }\end{array}$} & $\begin{array}{l}\text { Nab-paclitaxel } \\
\text { (Abraxane) }\end{array}$ & Chemotherapy & Paclitaxel & $\begin{array}{l}\text { Breast cancer; } \\
\text { non-small-cell lung } \\
\text { cancer; pancreatic } \\
\text { cancer }\end{array}$ & $\begin{array}{l}\text { Approved by } \\
\text { FDA (2005; } \\
\text { 2012; 2013) }\end{array}$ & - \\
\hline & & \multirow{2}{*}{$\begin{array}{l}\text { Nab-rapamycin } \\
\text { (ABI-009) }\end{array}$} & \multirow[t]{2}{*}{ Chemotherapy } & \multirow[t]{2}{*}{ Rapamycin } & PEComa & Phase II & NCT02494570 \\
\hline & & & & & $\begin{array}{l}\text { Advanced PEComa; } \\
\text { malignancy with } \\
\text { relevant genetic } \\
\text { mutations or mTOR } \\
\text { pathway activation }\end{array}$ & $\begin{array}{l}\text { Expanded } \\
\text { Access }\end{array}$ & NCT03817515 \\
\hline & & \multirow{3}{*}{$\begin{array}{l}\text { Aldoxorubicin } \\
\text { (DOXO-EMCH, } \\
\text { INNO-206) }\end{array}$} & \multirow[t]{3}{*}{ Chemotherapy } & \multirow[t]{3}{*}{ Doxorubicin } & $\begin{array}{l}\text { Advanced solid } \\
\text { tumour }\end{array}$ & Phase I & NCT01673438 \\
\hline & & & & & Glioblastoma & Phase II & NCT02014844 \\
\hline & & & & & $\begin{array}{l}\text { Metastatic, locally } \\
\text { advanced or } \\
\text { unresectable soft } \\
\text { tissue sarcoma }\end{array}$ & Phase III & NCT02049905 \\
\hline \multirow{2}{*}{ Nucleic acid } & \multirow{2}{*}{$\begin{array}{l}\text { Spherical } \\
\text { nucleic acid }\end{array}$} & \multirow[t]{2}{*}{$\begin{array}{l}\text { Cavrotolimod } \\
\text { (AST-008) }\end{array}$} & Immunotherapy & $\begin{array}{l}\text { AST-008 (toll-like } \\
\text { receptor } 9 \text { agonist } \\
\text { oligonucleotide) }\end{array}$ & $\begin{array}{l}\text { Healthy volunteer } \\
\text { study }\end{array}$ & Phase I & NCT03086278 \\
\hline & & & $\begin{array}{l}\text { Immunotherapy } \\
\text { in combination } \\
\text { with } \\
\text { chemotherapy }\end{array}$ & $\begin{array}{l}\text { AST-008, } \\
\text { Pembrolizumab, } \\
\text { Cemiplimab }\end{array}$ & $\begin{array}{l}\text { Solid tumours; } \\
\text { melanoma; head and } \\
\text { neck squamous cell } \\
\text { carcinoma; cutaneous } \\
\text { squamous cell } \\
\text { carcinoma; Merkel } \\
\text { cell carcinoma }\end{array}$ & Phase lb/ll & NCT03684785 \\
\hline
\end{tabular}

mTOR, mechanistic target of rapamycin; PEComa, perivascular epithelioid cell tumours; PEG, polyethylene glycol.

countermeasures that can only be achieved with multifunctionality.

Cancer progression is a collective result of numerous pathological events ${ }^{14}$, which are often not tumourspecific but involve normal physiological processes distorted by the disease. Moreover, tumour cells are highly heterogeneous and have high mutation rates, resulting in pathological differences between different types of cancers, individuals and intratumoural regions. To address this heterogeneity, nanostructure-based combinatorial or multiple-target therapies are being explored. For example, coupling tumour-cell-targeted methods with strategies that modulate the tumour microenvironment (TME) (BOX 1) enables the therapy to be tailored to distinct tumour characteristics. In highly fibrotic tumours, cancer-associated fibroblasts (CAFs) or stellate cells can be co-targeted, in addition to cancer cells, to inhibit the secretion of growth factors and pro-inflammatory cytokines and to distribute nanostructure-delivered therapeutics across the dense stroma $^{15-17}$. Moreover, the recurrence of tumours after vasculature occlusion by targeted delivery of thrombin 
can be considerably decreased by co-delivery of doxorubicin, probably because the chemotherapeutic helps to eradicate tumour regions that are less populated by blood vessels ${ }^{18}$.

Although the development of a tumour-specific drug is extremely difficult, optimizing the pharmacokinetics and biodistribution of a less specific drug can substantially increase its efficacy. Indeed, the capacity of tumour-targeted delivery, either through passive targeting based on the enhanced permeability and retention (EPR) effect, active targeting or stimuli-responsive drug release, has been considered a key advantage of nanomedicines in cancer treatment ${ }^{2}$. However, compared with conventional drug formulations, the in vivo behaviour

\section{Box 1 | Tumour microenvironment targets for multifunctional nanostructures}

\section{Stroma and fibroblasts}

In the tumour microenvironment (TME), quiescent stromal cells become proliferative and highly secretory, resulting in a dense extracellular matrix (ECM), which acts as an intratumoural barrier against drug penetration. Moreover, ECM components, cytokines and chemokines secreted by activated cancer-associated fibroblasts and mesenchymal stromal cells promote tumour cell proliferation, invasion and acquisition of drug resistance ${ }^{15}$. Stromal cell proteins (such as fibroblast activation protein $\alpha$ ) and ECM components (especially ECM enzymes) can be used as targets for delivery or selective drug release ${ }^{16}$. Drugs targeting stromal abnormalities can be coupled with TME-targeting strategies to reverse the activation of stromal cells, inhibit protumoural signalling, or reduce the synthesis and secretion of fibrotic ECM components such as hyaluronan and collagen ${ }^{15,17}$.

\section{Blood vessels}

In many (but not all) ${ }^{241}$ solid tumours, angiogenesis is shifted towards the pro-angiogenic state, leading to a tortuous, interrupted vasculature network, which limits drug perfusion. Vascular endothelial cells conditioned by the TME actively participate in T-cell exclusion or metastasis, and the pro-coagulative state of tumours leads to the activation of platelets, which can promote tumour invasion ${ }^{242-244}$. Endothelial receptors and tumour-associated microthrombi can be targeted to localize nanostructures to the tumour vasculature ${ }^{40}$. Drugs can be released into the TME to modulate intratumour angiogenic signalling (for example, the vascular endothelial growth factor (VEGF) pathway) or to neutralize excessive pro-angiogenic factors, which are generated in response to cytotoxic therapies ${ }^{245}$.

\section{Immune cells}

Most immune-cell-targeted nanotherapies aim to enhance the anticancer cytotoxic T-cell response. Treatments that directly target T cells using immune checkpoint (cytotoxic T-lymphocyte-associated protein 4 (CTLA-4) or programmed cell death protein 1 (PD-1)) inhibitors have shown promise against hot tumours, in which active T cells are able to infiltrate but are functionally blocked by immune checkpoint signalling ${ }^{246}$. In cold tumours, T cells are not activated, owing to the presence of immunosuppressive immune cells, which can be targeted to enhance T-cell recruitment. Nanovaccines that deliver tumour antigens and immunostimulatory agents to dendritic cells can improve their maturation and antigen presentation ${ }^{247,248}$. Reprogramming of protumoural macrophages to antitumoural phenotypes can revive their antitumour cytotoxic activity and boost T-cell responses ${ }^{50,51}$. Natural killer (NK) cells spontaneously attack cancer cells without requiring antigen sensitization. Targeting NK cells with cytokines (for example, IL-15) or antibodies that induce stimulation and expansion provides an alternative to T-cell-based immunotherapy ${ }^{249}$.

\section{Lymphatic vessels}

Solid tumours lack functional lymphatic drainage; however, lymphatic vessels are important highways for metastasizing cancer cells. Indeed, high levels of lymphangiogenesis in tumours correlate with poor outcome ${ }^{250,251}$. Tumour-associated lymphatic endothelial cells overexpress immunosuppressive molecules, such as PD-L1, but also promote dendritic cell migration into lymph nodes, associated with high infiltration of naive T cells ${ }^{250}$. Tumoural lymphangiogenesis can be modulated by blocking VEGFR3, the receptor of VEGF-C, a tumour-cell-secreted lymphangiogenic factor $^{251}$. However, targeting ligands for lymphatic vessels remain limited (with the example of the peptide LyP-1 ${ }^{252}$ ). and fate of multifunctional nanostructures are more complex, which can make them particularly prone to inconsistencies across patients and between preclinical models and clinical applications ${ }^{19}$.

The properties of nanostructures are instantly affected when entering the body by interactions at the nano-bio interface. These interactions can lead to instabilities, such as particle aggregation, decomposition, loss of functional units or release of hazardous species, which greatly affect delivery efficiency and biosafety ${ }^{20}$. Contact with physiological fluids also leads to cloaking of the nanostructure with serum proteins (the protein corona), potentially shielding surface functionalities (such as targeting ligands) and interfering with drug release $^{21}$. Antifouling modifications, such as coating with poly(ethylene glycol) (PEG), are often applied to reduce nanoparticle-protein interactions. Alternatives based on biomolecules, especially proteins and peptides, have also been reported ${ }^{22,23}$. In addition, interference of the protein corona with surface functionalities depends on the functionalization technique; for example, antibodies pre-adsorbed onto polymeric nanoparticles retain their targeting ability better than chemically conjugated molecules, perhaps owing to more robust surface coverage ${ }^{24}$. By tailoring nanosurface modifications, the protein corona composition can be manipulated and exploited to increase circulation, reduce toxicity or improve targeting $^{21,25}$. For example, Onpattro, a commercially available small interfering RNA (siRNA) formulation, recruits the liver-targeting protein, apolipoprotein $\mathrm{E}$, onto the cholesterol-containing lipid nanoparticle surface in vivo ${ }^{26}$. Short peptides have also been used to manipulate the binding modes of serum apolipoproteins on the nanoparticle surface to improve delivery across the blood-brain barrier ${ }^{27}$. Although interference from non-specific adsorption needs to be carefully characterized $^{28}$, this approach suggested the possibility of modulating corona proteins more precisely and using them as additional functionality.

In the bloodstream, phagocytes readily adhere to protein corona-coated nanostructures through specific receptors, which leads to removal of the nanostructures from the circulation by the reticuloendothelial system (RES ${ }^{29}$. Thus, antifouling coatings, such as PEG, are commonly used to prolong the circulation time of nanostructures. Nonetheless, PEG modification only partly reduces immunogenicity, and the stimulation of PEG-binding antibodies has raised safety concerns ${ }^{30,31}$. More active antifouling strategies include surface decoration with moieties based on CD $47^{32,33}$, a 'self-marker' protein that inhibits phagocytosis, or with extracted blood cell membranes (displaying CD47 on their surface $)^{31,34}$, to help nanomaterials to evade clearance by phagocytes. In mouse models, these methods have demonstrated superior stealth properties compared with PEGylation $^{31,32,34}$. However, corona-mediated clearance can also benefit macrophage- or RES organ-targeted delivery. For example, by precisely alternating the charge of cationic liposomes, they can be selectively directed to the liver and spleen to deliver genes ${ }^{35}$.

To reach tumour tissue, nanosystems first need to extravasate from the circulation ${ }^{1,36}$, which has long been 


\section{Box 2 |Administration routes for cancer-targeting nanostructures}

Intravenous administration is characterized by a rapid therapeutic response, high drug bioavailability and high administration control. Structurally complex and biologically unstable nanosystems are often not suitable for gastrointestinal absorption or intramuscular injection, and thus intravenous injection is often the method of choice. In addition, anti-metastasis therapies require systemic drug administration. However, intravenous administration suffers from rapid drug clearance, undesired tissue distribution and accumulation, and subsequent adverse side effects.

Oral delivery benefits from good patient compliance and may provide easy access for gastrointestinal tract targeting. However, the harsh and complex environment in the gastrointestinal tract (high pH variation, metabolic enzymes, mucus-mediated clearance) provides challenges for nanostructures. Mucus-adhering and endothelium-penetrating elements are usually required to increase intestinal adsorption and penetration of nanomaterials.

Inhalation and intranasal administration allow access to the respiratory tract (for example, for the treatment of lung cancer) and enable sustained and less invasive targeting, as compared with intravenous injection. Nanostructures also require mucus-adhering functionalities to enhance mucus adhesion and penetration (as for other mucosal routes, such as vaginal delivery $)^{253}$. However, inhaled or intranasally delivered drugs can also be systemically distributed; indeed, intranasally delivered drugs have been found to accumulate in the central nervous system ${ }^{253}$.

Direct injection and infusion into tissues adjacent to the tumour site is an option if tumours are readily accessible. Local administration routes bypass many biological barriers (for example, drugs administered into the brain bypass the blood-brain barrier), but tissue penetration is still required. Approaches to enhance nanomaterial penetration and retention in the dermis and subcutaneous tissue remain limited ${ }^{254}$. Locally injected small particles are prone to rapid lymphatic drainage, and thus subcutaneous administration can deliver nanoparticles to lymph nodes and immune cells that reside in the lymphatic system.

Each route of administration leads to a distinct drug distribution and distinct patterns of adverse effects. Safety issues related to non-intravenous routes, such as inhalation or skin contact, need to be considered ${ }^{255}$. Systematic studies of route-specific toxicities in the context of cancer nanomedicine will be essential.

thought to be enabled by leaky tumour blood vasculature. However, the EPR effect greatly varies between tumours and even between different regions of the same tumour, and its clinical significance remains controversial ${ }^{37-39}$. Multifunctional nanoparticles can actively increase intratumoural extravasation by creating local hypertension, causing vascular relaxation or inducing additional damage in tumour blood vessels. Such strategies may partly address the problem of EPR heterogeneity ${ }^{38,40}$, but stratification of patients and imaging-guided personalized medicine will prove essential to tailor nanomaterial-based therapeutic strategies ${ }^{1,2,41}$.

In addition, extravasation efficiency is affected by the nanomaterial properties; for example, in flowing blood, spherical nanoparticles tend to stay close to the middle of the vessel, whereas rod- or plate-shaped structures are more likely to drift towards the vessel walls and exhibit strong interactions with endothelial cells, owing to the larger contact area ${ }^{36,42}$. The surface softness of extracellular vesicles substantially impacts extravasation and cellular uptake in vivo ${ }^{43}$. Endothelial adhesion, and thus extravasation, can be enhanced by coating nanoparticles with cell membranes derived from leucocytes ${ }^{44}$. Extravasation is generally thought to occur by paracellular leaking; however, other mechanisms, such as active transport by endothelial cells ${ }^{39,45}$, also play a role, and are receiving attention in terms of nanosystem design and targeting $^{46,47}$.
Most anticancer nanoformulations are designed for intravenous use. Of note, challenges posed by biological barriers during nanoparticle transport are related to the administration route; for example, multifunctional strategies have not yet addressed the problem of intravenously injected nanomaterial accumulation in the liver, which can help to reduce the toxicity of delivered drugs to other tissues and improve the overall tolerance ${ }^{12}$, but remains a problem for efficient drug delivery to the tumour. The administration route determines the biodistribution pattern of drugs, and routes alternative to intravenous injection with corresponding targeting strategies are explored for specific cancers (BOX 2). Intradermal and subcutaneous injection, for example, may be particularly useful to target the lymphatic system for tumour immunization ${ }^{48}$, whereas orally delivered nanoparticles can target colon cancer ${ }^{49}$.

The tumour and the TME also provide a barrier to nanostructure-based drug delivery. High interstitial fluid pressure and desmoplastic stroma limit the penetration of nanostructures ${ }^{6,36}$. Moreover, deeper tumour regions are less populated by blood vessels. In addition, nanoparticles can be captured by tumour-associated macrophages (TAMs) instead of tumour cells following extravasation ${ }^{41}$. These intratumoural complications have motivated the development of TME-targeting strategies (BOX 1), exploiting potential targets that are more accessible than tumour cells. Indeed, TME-related targets, such as the fibroblast activation protein on $\mathrm{CAFs}^{16}$, fibrin-fibronectin complex in tumour blood vessels ${ }^{18}$ or TAMs ${ }^{50,51}$, have already been applied for nanoparticle delivery. For therapies that require intracellular delivery (for example, gene delivery and antigen delivery), cellular uptake and endosomal escape are needed. However, unlike hydrophobic small drugs, nanoparticles cannot passively diffuse across the plasma membrane. Therefore, membrane-protein-targeted ligands, cell-penetrating peptides or fusogenic materials are used to promote cellular uptake ${ }^{5,52}$.

\section{Multifunctional nanostructures}

Multifunctional nanoformulations enable multitargeted and/or combinatorial therapy, theranostics, tumour-specific drug delivery, bypassing of biological barriers and improved pharmaceutical properties (for example, pharmacokinetics, stability, biodegradation and biocompatibility), as compared with free drugs (TABLE 2). A multifunctional nanoplatform for cancer therapy typically consists of a structural framework, functional units and loaded cargo(es), which may also be considered a functional element (FIG. 1). To simplify fabrication and reduce unwanted side effects, the framework can also serve as functional element. For example, peptide drugs can be modified and self-assembled into nanosized particles to improve their pharmacokinetic profile. Biomolecules have particularly useful properties as backbone building blocks and can act as versatile substrates for multifunctionalization.

Polysaccharide-based nanostructures. Polysaccharides are biopolymers consisting of monosaccharide units linked together by glycosidic bonds. Polysaccharidebased materials provide a range of functional groups and 
Table 2 | Anticancer functionalities of biomolecule-based nanostructures

\begin{tabular}{|c|c|c|c|}
\hline Anticancer functions & Functional components & Aims and advantages & Refs \\
\hline \multicolumn{4}{|l|}{ Bypassing biological barriers } \\
\hline $\begin{array}{l}\text { Reduced immune recognition and } \\
\text { RES clearance }\end{array}$ & $\begin{array}{l}\text { Low-protein-adsorption peptides or } \\
\text { polysaccharides; anti-phagocytosis peptides; } \\
\text { blood cell membranes; albumin; DNA } \\
\text { tetrahedron; ferritin }\end{array}$ & $\begin{array}{l}\text { Prolonged circulation time and evasion of } \\
\text { liver and spleen capture }\end{array}$ & $\begin{array}{r}34,90,175,177, \\
233,234\end{array}$ \\
\hline Enhanced extravasation & $\begin{array}{l}\text { Albumin; leucocyte membranes; rod-like } \\
\text { nanostructures }\end{array}$ & $\begin{array}{l}\text { High intratumoural delivery efficiency; high } \\
\text { exposure of functional elements to molecular } \\
\text { targets; microenvironmental trigger }\end{array}$ & $44,146,175$ \\
\hline $\begin{array}{l}\text { Enhanced tumour tissue } \\
\text { penetration }\end{array}$ & $\begin{array}{l}\text { Albumin; stroma-targeted ligands; } \\
\text { cell-penetrating peptides }\end{array}$ & $\begin{array}{l}\text { Treatment of less accessible tumour cells } \\
\text { deep within tumour tissue }\end{array}$ & 16,177 \\
\hline $\begin{array}{l}\text { Delivery across the blood-brain } \\
\text { barrier }\end{array}$ & $\begin{array}{l}\text { Albumin; DNA tetrahedron; brain-targeted } \\
\text { peptides }\end{array}$ & $\begin{array}{l}\text { Treatment of brain tumours with } \\
\text { intravenously delivered formulations }\end{array}$ & 27,175 \\
\hline $\begin{array}{l}\text { Delivery across the air-blood } \\
\text { barrier }\end{array}$ & Albumin & $\begin{array}{l}\text { Facilitated pulmonary delivery of agents } \\
\text { to treat lung cancer }\end{array}$ & 178 \\
\hline $\begin{array}{l}\text { Delivery across the intestinal } \\
\text { endothelium }\end{array}$ & $\begin{array}{l}\text { Chitosan surface coating; } \\
\text { endothelium-penetrating ligands }\end{array}$ & $\begin{array}{l}\text { Treatment of intestinal gut tumours; } \\
\text { orally administered chemotherapy }\end{array}$ & $49,61,65,235$ \\
\hline \multicolumn{4}{|l|}{ Tumour-targeted delivery } \\
\hline Ligand-receptor recognition & Aptamers; albumin; peptides; folic acid; ferritin & \multirow{2}{*}{$\begin{array}{l}\text { Selective drug delivery to and accumulation } \\
\text { in specific tissues, cells or subcellular } \\
\text { organelles }\end{array}$} & $\begin{array}{l}18,58,67,88,90,91,99, \\
150,177,179,233,234\end{array}$ \\
\hline Protein corona manipulation & Adsorbed serum proteins & & \\
\hline \multicolumn{4}{|l|}{ Stimuli responsiveness } \\
\hline $\begin{array}{l}\text { Stimuli-triggered decomposition of } \\
\text { nanostructures }\end{array}$ & $\begin{array}{l}\text { Protonable polysaccharides and peptides }(\mathrm{pH}) \text {; } \\
\text { enzyme-cleavable peptides (tumour-specific } \\
\text { enzymes); temperature-sensitive peptides (heat); } \\
\text { disulfide bonds ( } \mathrm{GSH}) \text {; irradiation-sensitive } \\
\text { bonds (external light or radioactive irradiation); } \\
\text { DNA nanoflower }\left(\mathrm{H}_{2} \mathrm{O}_{2}\right) \text {; DNA nanohydrogel } \\
\text { (pH); spherical nucleic acid (GSH); DNA origami } \\
\text { (GSH); quantum dot DNA hydrogels (DNase); } \\
\text { DNA coordination polymer (pH); albumin }\left(\mathrm{H}_{2} \mathrm{O}_{2}\right) \text {; } \\
\text { ferritin (pH); GroEL (ATP) }\end{array}$ & $\begin{array}{l}\text { Rapid and complete drug release from } \\
\text { otherwise stable nanostructures; selective } \\
\text { drug release in desired location }\end{array}$ & $\begin{array}{l}16,59,63,93, \\
157,216,234\end{array}$ \\
\hline $\begin{array}{l}\text { Stimuli-triggered (in situ) } \\
\text { self-assembly (including gel } \\
\text { formation) of nanostructures }\end{array}$ & Albumin; stimuli-responsive peptides & $\begin{array}{l}\text { Delivery of nanostructures not suitable for } \\
\text { administration in the assembled form owing } \\
\text { to size, shape or mechanical properties }\end{array}$ & $148,150,179$ \\
\hline $\begin{array}{l}\text { Stimuli-triggered shape } \\
\text { transformation of nanostructures }\end{array}$ & $\begin{array}{l}\text { Peptide assembly; DNA origami (protein); DNA } \\
\text { nanosuitcase (mRNA); DNA clamp (antibody); } \\
\text { GroEL (ATP) }\end{array}$ & $\begin{array}{l}\text { Rapid and complete drug release from } \\
\text { otherwise stable nanostructures; selective } \\
\text { drug release in desired location }\end{array}$ & $\begin{array}{r}3,95,96,99, \\
150,216,217\end{array}$ \\
\hline $\begin{array}{l}\text { Stimuli-triggered activation and } \\
\text { shedding of targeting, therapeutic } \\
\text { or imaging functionality of } \\
\text { nanostructures }\end{array}$ & $\begin{array}{l}\text { Aptamer; ferritin; enzyme-cleavable peptides; } \\
\text { breakable bonds }\end{array}$ & $\begin{array}{l}\text { Minimization of non-specific nano-bio } \\
\text { interactions; imaging signal detection } \\
\text { during delivery; selective drug release in } \\
\text { desired location }\end{array}$ & $55,88,191$ \\
\hline $\begin{array}{l}\text { Stimuli-triggered surface } \\
\text { transformation (for example, charge } \\
\text { reverse) of nanostructures }\end{array}$ & Protonable peptides and polysaccharides & $\begin{array}{l}\text { Selective alteration of surface charge or } \\
\text { functional groups at the tumour site to } \\
\text { facilitate cell penetration, lysosome escape } \\
\text { or other biological effects }\end{array}$ & \\
\hline \multicolumn{4}{|l|}{ Anticancer drug building blocks } \\
\hline Targeting ligand & Antibodies; therapeutic peptides and aptamers & \multirow{2}{*}{$\begin{array}{l}\text { Low metabolic burden and minimal adverse } \\
\text { effects }\end{array}$} & \multirow[t]{2}{*}{$127,157,196,197,202$} \\
\hline Self-assembly material & $\begin{array}{l}\text { Therapeutic peptides and aptamers, CpG DNA } \\
\text { nanococoon, DNA-RNA nanocapsules, plant } \\
\text { viral nanoparticles, protozoa protein, E2 subunit } \\
\text { of pyruvate dehydrogenase }\end{array}$ & & \\
\hline \multicolumn{4}{|l|}{ Combinatorial therapy } \\
\hline Multidrug loading & $\begin{array}{l}\text { Albumin; DNA origami; amphiphilic } \\
\text { polysaccharides; amphiphilic peptides }\end{array}$ & \multirow{3}{*}{$\begin{array}{l}\text { Combined advantages of multiple drugs or } \\
\text { therapeutic modalities; mechanism-based } \\
\text { cooperation to improve tumour sensitivity to } \\
\text { other treatment and to overcome resistance } \\
\text { or to prevent recurrence }\end{array}$} & $18,57,63,175,178$ \\
\hline Cooperation with immunotherapy & $\begin{array}{l}\text { Immune cell-targeting ligands; DNA } \\
\text { nanohydrogel; DNA-RNA nanocapsules; } \\
\text { ferritin; hepatitis B core protein }\end{array}$ & & $127,185,192$ \\
\hline $\begin{array}{l}\text { Cooperation with phototherapy or } \\
\text { radiotherapy }\end{array}$ & $\begin{array}{l}\text { DNA coordination polymer; albumin; } \\
\text { nanostructures containing sensitizer or } \\
\text { anti-angiogenetic drug }\end{array}$ & & $67,93,176$ \\
\hline
\end{tabular}


Table 2 (cont.) | Anticancer functionalities of biomolecule-based nanostructures

\begin{tabular}{lll}
\hline Anticancer functions & Functional components & Aims and advantages \\
\hline $\begin{array}{l}\text { Theranostics } \\
\begin{array}{l}\text { Simultaneous imaging and } \\
\text { treatment of tumour tissue }\end{array}\end{array}$ & $\begin{array}{l}\text { Nanostructures containing fluorophores; } \\
\text { magnetic nanoparticles or other imaging } \\
\text { agents }\end{array}$ & $\begin{array}{l}\text { Simultaneous diagnosis of hidden tumours } \\
\text { or small metastasis }\end{array}$ \\
$\begin{array}{l}\text { Real-time monitoring of drug } \\
\text { release and activity }\end{array}$ & $\begin{array}{l}\text { Separable fluorophore-quencher pairs; } \\
\text { fluorescent molecules with stimuli-dependent } \\
\text { emission properties }\end{array}$ & $\begin{array}{l}\text { Monitoring of in vivo activity of } \\
\text { nanostructures or tumoural response to } \\
\text { delivered drug }\end{array}$ \\
\hline
\end{tabular}

show good biodegradability, stability, long-term durability and, in general, low immunogenicity ${ }^{8,53}$. Many polysaccharides, such as cellulose, starch, alginate and chitosan, are abundant in nature and can be extracted from renewable sources ${ }^{5,55}$. These characteristics make polysaccharides interesting alternatives to synthetic polymers in nanomedicine. Almost all known types of polysaccharides have been explored for cancer-targeted drug delivery or theranostics ${ }^{8,54,56}$.

Long-chained polysaccharides can be fabricated into diverse nanostructures for drug loading. Polysaccharidebased building blocks are commonly assembled into micelles, and anticancer drugs can then either be encapsulated or linked to the saccharide chain. Grafting with polymers, hydrophobic tails or protonable groups allows tuning of solubility and amphilicity. Saccharides containing primary amines (for example, chitosan), amides (for example, chitosan, heparin and hyaluronan), carboxyls (for example, heparin and hyaluronan) or primary hydroxyl groups (for example, hyaluronan and cyclodextrin) can be covalently modified, enabling the formation of amide bonds or ester bonds, and the glucosidic ring can also be opened by oxidation ${ }^{8}$. Targeting ligands ${ }^{57,58}$, stimuli-sensitive elements (most commonly redox-responsive disulfide bonds $)^{55,59,60}$ or other biologically active entities can thus be integrated into the biopolymer backbone ${ }^{61}$. Certain saccharides also allow additional modes for material-drug interaction; for example, negatively charged drugs, such as nucleic acids, can be electrostatically attached to protonable amine groups of chitosan, enabling gene delivery. NLG207, a topoisomerase inhibitor nanoformulation currently in phase I/II trials (TABLE 1), consists of $\beta$-cyclodextrin-PEG copolymer chains that self-assemble into nanoparticles through guest-host recognition between cyclodextrin and the conjugated active component camptothecin, which improves the solubility and tumour exposure of the otherwise hydrophobic drug ${ }^{62}$.

Some polysaccharides have been exploited for their intrinsic targeting and therapeutic functions. Hyaluronic acid is a major component of the extracellular matrix and non-immunogenic. Its specific receptor CD44 is overexpressed in many types of cancer $^{8,56}$, and thus hyaluronic-acid-based nanostructures can be applied for CD44-mediated targeting and cell uptake $e^{60,63,64}$. The protonatable amine groups of chitosan facilitate intestinal mucus adhesion and penetration, and chitosan nanoparticles can therefore be used for oral chemotherapy ${ }^{61,65}$. The biological properties of other polysaccharide nanomaterials, however, have been less explored. For example, fucoidan, a fucose-rich sulfated polysaccharide derived from brown seaweed, specifically binds to P-selectin, an adhesion protein that facilitates metastasis by mediating the interactions between tumour cells and platelets or blood vessel endothelia ${ }^{66}$. Fucoidan-containing nanoparticles have been shown to be effective P-selectin-targeted drug carriers ${ }^{67,68}$; however, fucoidan is difficult to purify, and its monosaccharide composition, molecular weight and structural characteristics vary depending on the source and purification method, which may limit rigorous study and clinical translation $^{66}$.

The clinical translation of polysaccharide nanostructures remains difficult, because many polysaccharides are derived from natural products. Depending on the source material and processing method, material parameters such as monosaccharide composition, glycosidic bond type, charge density, molecular weight and biomacromolecule contamination can differ, which can cause variations in biological effects ${ }^{8,66}$. For example, alginate, a seaweed-derived carbohydrate, is usually regarded as biosafe; by contrast, alginate with high mannuronate, rather than guluronate, content can induce the production of inflammatory cytokines in vitro ${ }^{69}$. For the majority of polysaccharide-based materials, systematic in vivo data are limited, and it is not known how the abovementioned parameters affect biomedical performance (for example, immunogenicity, pharmacokinetics, cellular uptake and biodegradation profile), impeding clinical translation. Moreover, the impacts of material characteristics on delivery efficiency are complex to decipher, leading to often inconsistent results. For example, the correlation of molecular weight and efficiency of chitosan as gene carrier has been controversial; it has been suggested that nanoparticles fabricated with long chitosan chains have a disadvantage in terms of RNA release, but promote cellular uptake and endosomal escape ${ }^{64}$. However, superiority of long-chain chitosan was observed only for mRNA transfection and not for siRNA silencing.

The potential advantage of polysaccharides as economic and renewable biomass remains underexplored in cancer nanomedicine. Polysaccharides in multifunctional nanostructures have mostly served as building blocks thus far and not as effectors of biological actions. Identifying their potential to trigger biological actions, in comparison with mainstream platforms such as liposomes and polymeric particles, would be of value. In any case, clinical translation of polysaccharidebased nanostructures will require systematic in vivo 
characterization and the development of methods for scalable fabrication and quality control.

Nucleic-acid-based nanostructures. Nucleic acids (that is, DNA and RNA) are natural building blocks that permit a bottom-up approach of molecular self-assembly enabled by highly stringent base pairing. They offer programmable molecular interactions and provide an almost unlimited number of sequences that can be used for the fabrication of defined nanostructures ${ }^{11,70}$.
They can be chemically fabricated in high yield and with low batch-to-batch variation in quality. In addition, negatively charged phosphate backbones or nucleobases that can chelate metal ions and form $\pi$-stacking can be modified to optimize pharmacokinetics and function $^{71}$. By introducing biological tags and stimuli-responsive units into DNA or RNA architectures, the features of nucleic acids can be extended far beyond their natural genetic roles. These attributes can be exploited for cancer targeting and therapy.

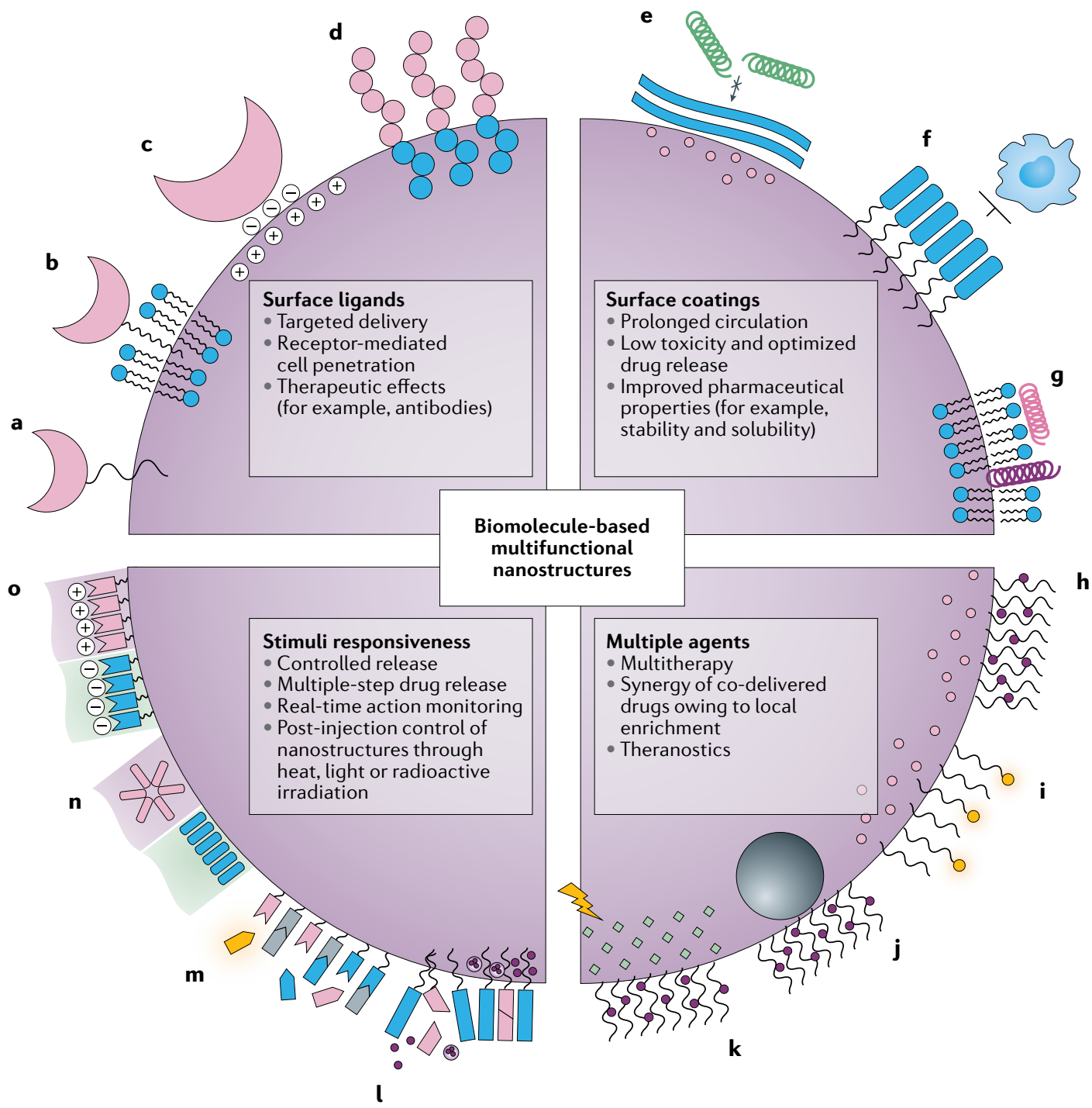

Fig. 1 | Functional elements in antitumour multifunctional nanomedicines. a-d | Surface ligands recognize tumourassociated molecules and epitopes for tumour targeting, penetration and therapy. Ligands can be attached through chemical conjunction (part a), hydrophobic interaction (part b) or electrostatic adsorption onto the nanoparticle surface (part c), or integrated as a functional motif into a peptide- or nucleic-acid-based molecular design (part d). e-g| Surface coatings shield the internal structure from non-specific nano-bio interactions and prevent early clearance during transport and distribution. e | Low protein adsorption on bioinert materials. $\mathbf{f} \mid$ Anti-phagocytosis molecules. $\mathbf{g} \mid$ Blood-cell-derived membrane camouflage. $\mathbf{h}-\mathbf{k}$ |Combination therapy or theranostic designs may be coupled with antitumour drugs. $\mathbf{h} \mid$ Multiple drug loading. $\mathbf{i} \mid$ Real-time imaging. $\mathbf{j} \mid$ Magnetic resonance steering. $\mathbf{k} \mid$ Phototherapy. $\mathbf{l}-\mathbf{o} \mid$ Stimuli-responsive elements. | Degradable building blocks trigger the disassembly of the nanocarrier to release its contents. $\mathbf{m}$ | Surface functionalities for selective activation by a stimulus. Bioactive components may be released or exposed on the nanostructure surface, which can be further monitored in real time by using quenchable or activable imaging agents. $\mathbf{n} \mid$ Environmental stimuli may induce structural transformation of nanostructures, often through stimuli-triggered re-assembly of the building units. $\mathbf{o}$ | Nanosurfaces may reverse their overall charge in specific microenvironmental niches. 
DNA and RNA nanotechnology has enabled the design of multidimensional nanostructures with high spatial accuracy for a range of applications ${ }^{72,73}$. DNA has high thermodynamic stability and $\mathrm{pH}$ tolerance, allowing DNA nanofabrication by precise molecular self-assembly ${ }^{71}$. Chemical modification strategies can also increase the stability of RNA, enabling RNA self-assembly into nanostructures ${ }^{74,75}$. For example, the $2^{\prime}-\mathrm{OH}$ of the ribose sugar moiety can be substituted with $2^{\prime}$-fluorine, $2^{\prime}$-O-methyl, $2^{\prime}$-O-methoxyethyl or $2^{\prime}$-amine to overcome the thermal and enzymatic susceptibility of RNA ${ }^{76}$. Moreover, the linkage between bases can be modified by phosphorothioate, boranophosphate, peptide nucleic acids and nucleic acid analogues, as well as by direct uracil or adenine modifications ${ }^{77,78}$. Complex nanoarchitectures based on nucleic acids can be engineered by various methods, including tile assembly ${ }^{79}$, origami techniques ${ }^{80}$, single-stranded tile methods ${ }^{81}$, rolling circle amplification ${ }^{82,83}$ and in silico design. Software packages, such as caDNAno, CanDo, oxDNA and ENERG-MD, have been widely used to design
DNA origamis ${ }^{84}$. However, accurate computational methods remain limited for RNA nanostructure design, although several computer programs have been developed, including NanoTiler, Mfold, RNA designer, Sfold and NUPACK ${ }^{76}$. Natural and custom-made nucleic acids can be engineered into 2D and 3D DNA, RNA, DNARNA and heterologous hybrid nanostructures, such as dynamic machines and supramolecular assemblies ${ }^{11,85}$. After decades of development, such nanostructures have become powerful tools in biomedical research (FIG. 2).

The most striking feature of nucleic-acid-based nanostructures is their spatial addressability, which can be achieved by modifying the sequence. Thus, functional molecules can be accurately integrated at specific locations in the nucleic acid structure to tailor the formulation for targeted delivery, smart cargo loading and release, simultaneous detection and visualization, and/or combinatorial therapeutic regimes ${ }^{11,84}$. Nucleic-acid-based assemblies can exploit the targeting modalities of DNA and RNA to engage cellular components. For example, aptamers, which are single-stranded

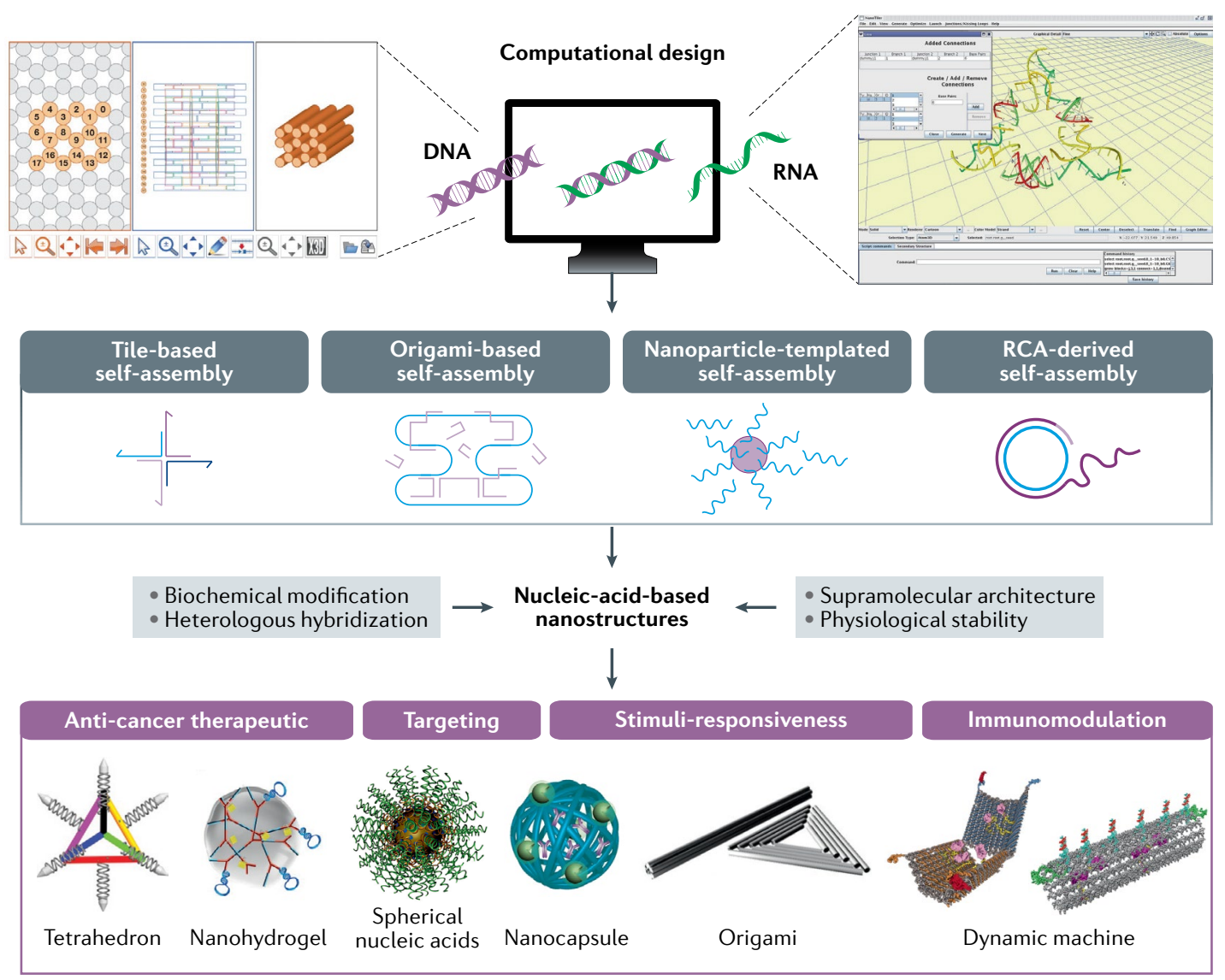

Fig. 2 | Nucleic-acid-based nanostructures for cancer therapy. Nucleic-acid-based nanostructures, including DNA, RNA, DNA-RNA and heterologous hybrid nanostructures, can be constructed with high spatial accuracy in different dimensions, using tile assembly, origami techniques, rolling circle amplification (RCA), nanoparticle-templated assembly and in silico design (for example, caDNAno, a software package for DNA origami design; NanoTiler, a computer program for RNA nanostructure design). These nanostructures, such as tetrahedrons ${ }^{91}$, nanohydrogels ${ }^{236}$, spherical structures ${ }^{237}$, nanocapsules ${ }^{83}$, origamis ${ }^{238}$ and dynamic machines ${ }^{3,99}$, can be applied as multifunctional anticancer systems. CaDNAno interface reprinted with permission from REF. ${ }^{239}$, OUP. NanoTiler program reprinted with permission from REF. ${ }^{240}$, Elsevier. Tetrahedron reprinted from REF. ${ }^{11}$, Springer Nature Limited. Spherical nucleic acids reprinted with permission from REF. ${ }^{237}$, ACS. Nanocapsule reprinted with permission from REF. ${ }^{83}$, Wiley. Origami reprinted with permission from REF. ${ }^{238}$, ACS. Dynamic machine reprinted with permission from REF., AAAS. 
DNA or RNA molecules (20-100 nucleotides in length) with defined secondary and tertiary structures, can interface with molecular targets with high specificity and affinity, comparable to antibodies ${ }^{86,87}$. By simply hybridizing or integrating aptamers with nucleic acid nanostructures, they can direct the specific recognition and binding of therapeutic agents by receptors expressed on malignant cells or to TME-specific biomarkers. The incorporation or self-assembly of microRNAs (miRNAs) or siRNAs into nucleic acid nanostructures enables gene delivery, and by adding cancer-cell-specific aptamers, non-specific adsorption and off-target effects can be prevented ${ }^{88}$. Therapeutic aptamers (for example, anti-human epidermal growth factor receptor 2 (HER2) aptamers) possess anticancer activity and enable tumour cell targeting, which improves efficacy and decreases the payload of nucleic acid nanostructures ${ }^{89}$.

Nanostructures based on nucleic acids can also be modified with other targeting ligands, such as antibodies, peptides and small molecules, through covalent or non-covalent linkage ${ }^{90,91}$. Additionally, nucleic acid nanostructures with certain hydrodynamic sizes $(10-200 \mathrm{~nm})$ and geometries (for example, triangular DNA origami) passively accumulate within tumour tissue, which improves targeting ${ }^{75,92}$. Efficient cargo delivery can also be achieved by incorporating environmentally responsive molecular switches in the nanostructures. For example, targetable properties of the intracellular environment and the TME (BOX 2) or external physical stimuli can be exploited for cancer targeting and therapy ${ }^{93,94}$.

Controllable drug release is a central aspect of targeted delivery in anticancer therapeutics, which can be accomplished by stimulus-responsive disassembly of nanocarriers. The computer-aided, controlled design of smart nanomachines or nanorobots based on nucleic acids allows the engineering of logic-gated therapeutics ${ }^{84}$. Cargo release kinetics at a specified target site can be customized by reconfiguration of nanostructures in response to dynamic changes in specific molecular switches or environmental cues. One of the earliest logic-gated DNA nanorobots is a hollow origami barrel that contains antibody Fab fragments against human leucocyte antigens ${ }^{3}$. A logical AND gate is programmed through a two-input system based on aptamer encoding; two different DNA aptamer 'locks' can only be opened by simultaneous recognition of both 'keys' on the target cell surface, which leads to opening of the nanostructures and release of an antibody cargo that inhibits cell growth. Dual lock-and-key strategies can also be incorporated into DNA nanodevices for conditional siRNA delivery and release in specific cellular environments, yielding high gene silencing efficiency and low off-target toxicity $^{88,95}$.

Reversible loading and release of nucleic acid cargo can also be achieved by a conformational change of an antibody-driven DNA-based nanomachine ${ }^{96}$. In particular, DNA origami nanorobots are explored for the precise delivery of therapeutics in vivo ${ }^{97,98}$. For example, a DNA origami nanorobot can precisely deliver a protease, which has strong adverse effects when administered in its free form, to tumour-associated blood vessels in tumour-bearing mice, with a high biosafety profile in both mice and Bama miniature pigs ${ }^{99}$. In this case, mechanical opening of the tubular DNA nanorobots is triggered by the specific binding of tumour endothelium-targeted DNA aptamers, leading to the release of thrombin from the inner cavity of the nanorobot, which causes intravascular thrombosis at the tumour site. Recently, a DNA machine communication network was developed that mimics the cascade reaction in the vertebrate adaptive immune system by stimulating immune cells upon the invasion of pathogens, demonstrating the great promise of DNA-based nanotherapeutics responding to sophisticated targets ${ }^{100}$. Taking advantage of the metastability of co-transcriptionally generated nucleic acid structures, a self-driven RNA nanomachine has been designed as an miRNA sensor, exploiting a mechanism beyond simple structural transformation $^{101}$. Although most proof-of-principle nucleic-acid-based nanomachines have not yet been thoroughly validated in vivo, they highlight the opportunities for dynamic nucleic-acid-based machines and nanorobots for targeted smart cancer treatment. Various other construction methods and applications of DNA machines have been explored ${ }^{84}$.

Although referred to as machines or robots, dynamic nucleic-acid-based nanostructures mainly rely on blood circulation for delivery and do not provide autonomous movement, as demonstrated in other micro- or nanostructures driven by exogenous power, such as magnetic fields, acoustic fields, electric fields and/or light energy, or by endogenous chemical reaction energy ${ }^{102}$. Such active transport mechanisms face several technical challenges; they require self-propelled motion; transport needs to be sustained by chemical or biological fuels; and navigation has to be controlled in the complex body environment, which shows irregular Brownian motion and can lead to interactions between the nanostructure and biological molecules ${ }^{103}$. The implementation of feedback mechanisms, such as automatic selection and optimal response chosen from a menu of available programs in response to a signal, will facilitate smart theranostics executed by nucleic-acid-based nanomachines.

DNA and RNA molecules are indispensable to essential immunological events, and thus have the ability to target and/or modulate immune cells ${ }^{104}$. Indeed, recombinant plasmid DNA and mRNA are promising vaccine platforms ${ }^{105,106}$, as demonstrated in COVID-19 vaccines. Currently, there are 22 mRNA-based and 15 DNA-based COVID-19 vaccine candidates in or entering clinical trials, including three mRNA-lipid nanoparticle vaccines encoding the SARS-CoV-2 spike protein or its receptor-binding domain ${ }^{107}$. The immunogenicity of a nucleic acid sequence can be completely reversed by a single nucleotide or base change, implying that precise control of the immune activity of nucleic acid nanoarchitectures is feasible ${ }^{70}$. Thus, the initial design of nucleic acid nanoarchitectures should take into account whether immune responses should be exploited (for example, for vaccines and immunotherapies) or mitigated (for example, for immunologically quiescent drug delivery).

The composition, structure and immunosuppressive properties of nucleic acid sequences need to be considered in the design of delivery vehicles based on nucleic 
acids to ensure suppression of immunogenicity during theranostic cargo delivery ${ }^{108-110}$. DNA nanostructures evoke weaker immune responses than DNA-RNA hybrids, which in turn are less immunostimulatory than their RNA counterparts ${ }^{104,111}$. Therefore, more DNA- than RNA-based nanostructures have been constructed as immunologically inert drug carriers for cancer treatment despite the fact that some RNA structures, such as the RNA four-way junction $(4 \mathrm{WJ}-\mathrm{X})$, do not elicit detectable immune stimulation in vivo ${ }^{112}$. As an alternative to decreasing the number of RNA strands, RNA strands can be modified with a $5^{\prime}$ end cap or certain RNA motifs can be embedded in the nanostructure in a certain orientation to attenuate immunogenicity ${ }^{113,114}$. Of note, drug-loading triangular DNA origami, a self-assembled DNA tetrahedral framework and 3D DNA nanotubes do not elicit detectable immunogenic responses in tumour-bearing mouse models ${ }^{91,99,115}$. The in vivo stability of nanostructures, which is typically enhanced by surface modifications, also affects the immune response, for example by altering the accessibility of vulnerable sites to nucleases ${ }^{116}$. Immunorecognition can be assessed by quantitative structure-activity relationship modelling, and preliminary results reveal that molecular weight, melting temperature and half-life largely dictate the immunomodulatory activity of nucleic acid nanostructures ${ }^{104,117,118}$. Therefore, a design road map is crucial for the precise tailoring of the immune processing of nucleic acid nanostructures in cancer therapy.

Nucleic acid nanostructures also offer the possibility of activating an immune response against tumours. Anticancer immune responses can be boosted by pathogen-associated molecular patterns, such as single-stranded DNA or double-stranded RNA, which are recognized by toll-like receptors (TLRs) on immune cells ${ }^{119}$. Unmethylated cytosine-phosphate-guanine oligodeoxynucleotides (CpG ODNs), for example, are processed by TLR9 and have thus been used as efficient adjuvants to induce strong immune responses in cancer immunotherapy ${ }^{120,121}$. Sequence-specific immunostimulation permits streamlined manufacturing of nucleic-acid-based nanostructures, without the need for complex multifunctional formulation. To maximize immune responses, an antigen can be formulated with a nucleic acid adjuvant through tailored engineering. For example, a DNA tetrahedron activates long-lasting immune responses in a TLR9-dependent manner in vertebrates, when co-assembled with $\mathrm{CpG}$ ODN adjuvants ${ }^{122,123}$. Spherical nucleic acids are also clinically promising nanostructures; here, high-density oligonucleotide nanoparticles precisely incorporate CpG ODNs for the co-delivery of tumour-associated antigens ${ }^{124,125}$. Using a high-throughput method, $\sim 1,000$ spherical nucleic acid architectures have been synthesized at the picomolar scale, and activation of the TLR9 pathway could be quantitatively screened by mass spectrometry ${ }^{118}$. Nucleic acid nanovaccines can create immunity in situations in which antitumour immune responses to other therapies are limited ${ }^{126}$. For example, a self-assembled hybrid DNA-RNA-peptide nanovaccine can deliver tandem CpG ODNs and signal transducer and activator of transcription 3 (STAT3) short hairpin RNA adjuvants, as well as tumour-specific peptide neoantigens, into antigen-presenting cells (APCs) in lymph nodes for synergistic cancer immunotherapy ${ }^{127}$. DNA and RNA aptamers have been used as antagonists of cytotoxic T-lymphocyte-associated protein 4 or programmed cell death protein 1 receptors to improve T-cell responses for cancer immunotherapy ${ }^{128,129}$. Thus, precisely incorporating aptamers into nucleic-acid-based nanostructures is a viable cancer immunotherapy strategy, enabling homogeneous synthesis of the nanostructures and modulation of various APCs. In addition, intracellular trafficking of nanostructures can be leveraged to trigger sensor-specific immune recognition in specific compartments by functional nucleic acid elements, facilitating cross-presentation of an antigen payload.

The clinical translation of nucleic-acid-based nanostructures requires the development of economically viable and robust methods for mass production, as well as stable modification of the functional nanostructures. The scalability challenge has been partially addressed by technological breakthroughs in producing highly purified nucleic acid strands and nanostructures in vivo. Macroscopic amounts of DNA origami can be produced by the simultaneous generation of long single-stranded DNA (ssDNA) scaffold strands and short staple strands in a litre-scale bioreactor culture of M13 bacteriophages. This approach reduces the production cost of DNA origami from $\sim$ US $\$ 200$ per milligram to around 20 cents $^{130}$. To improve chemical synthesis through adding of 2 '-protecting groups, single-stranded RNA can also be transcribed from gene-encoded DNA and folded into designed nanostructures inside Escherichia coli cells, similar to the mass production of recombinant proteins $^{131}$. Although large-scale synthesis at the kilogram level is expected to reduce the price of pharmaceutical oligonucleotides to a few dollars per milligram, the requirements of chemistry, manufacturing and controls. and of good manufacturing practices. will certainly increase production costs ${ }^{108}$.

Structural integrity is essential for the nanostructures to function in vivo ${ }^{76,132}$. DNA nanostructures can be stabilized through covalent crosslinking between strands, without the need for additional chemical modification or cofactors ${ }^{133}$. In addition, polymer-based (such as PEGylated oligolysines and dendritic oligonucleotides) or lipid-based coatings can be applied ${ }^{134-136}$. Certain structural elements, such as single-duplex edges, make 3D DNA origami more stable in physiological environments, as compared to close-packed bundles of helices ${ }^{137-139}$. To rigorously determine the structural integrity and pharmacokinetics of nucleic acid architectures in the highly heterogeneous TME, robust techniques, surpassing currently used fluorescent or radioactive labelling in terms of stability, spatiotemporal resolution and sensitivity, are needed at the molecular and in vivo levels. The interaction between nucleic-acid-based nanostructures and cellular DNA or RNA must also be kept at a minimum. Therefore, standards for the predictive and rational design of nanostructures are indispensable to achieve clinical translation. 
Peptide-based nanostructures. Peptides are perhaps the most extensively studied materials for selfassembly-based nanosystems $s^{9,140-142}$. Compared with base-pairing-guided assembly of nucleic acids, the self-assembling behaviour of peptide molecules is less programmable. However, with 20 different natural amino acids, peptides offer a broader range of customizable physicochemical properties by sequence-specific engineering, as compared with nucleic acids. Peptides can be easily synthesized and functionalized, and are usually more stable in storage than proteins and nucleic acids. Moreover, many peptide motifs bear distinct biological roles, particularly for molecular recognition and signal transduction ${ }^{143}$.

Peptide self-assembly is a synergistic result of non-covalent intermolecular forces, including hydrophobic, electrostatic, hydrogen, van der Waals and $\pi-\pi$ interactions. By manipulating these forces, either through sequence design or through additional functionalization, the peptides can be assembled into particles, fibres, tubes, sheets or $3 \mathrm{D}$ gels with various dimensions, morphologies and surface chemistries ${ }^{140}$. The assembly processes can also be co-mediated or assisted by other agents; for example, hydrophobic small molecule drugs can transform amphiphilic peptide nanofibres into spherical structures, owing to the formation of tight cores driven by drug-peptide hydrophobic interactions ${ }^{16}$. Inspired by the assisted folding process of proteins, folding-assisting enzymes have been applied to form $\alpha$-helical peptide assemblies with very short peptides, which is not feasible without the help of enzymes $^{144}$.

A thorough understanding of peptide supramolecular chemistry is important for the accurate tailoring of their biological performance. For example, a modular system of nanoscale peptide cages modified with short charged peptides can promote endocytosis in vitro by controlling the charge of the cages ${ }^{145}$. A 'patched' hollow peptide tube can be engineered by mixing two types of peptides, which individually self-assemble into tubular and spherical structures, respectively. The hollow tube is sealed on both ends by half-spheres ${ }^{146}$, and can be applied as a drug-encapsulating vehicle, enabling extravasation into tumour tissue, comparable to other rod-like nanocarriers.

Minor changes in the forces governing peptide assembly can lead to substantial structural transformations ${ }^{140}$, rendering peptide-based materials particularly versatile as stimuli-sensitive devices. Peptides can also alter their functionality in response to tumour-related stimuli; for example, $\mathrm{pH}$-sensitive cell-penetrating peptides ${ }^{147}$ are mainly internalized in the slightly acidic TME and not in healthy tissue. In a 'reverse' stimuli-responsive strategy, disassembled ordered structures, free peptides or small nanoassemblies aggregate at the tumour site to form larger nanostructures for sustained drug release, real-time imaging or to induce nanomaterial-mediated cytotoxicity. This approach reduces potential adverse side effects owing to rapid clearance of off-target free peptides $^{148,149}$. For example, a hydrophobic photosensitizer (Purpurin18)-linked tumour-homing peptide is specifically cleaved by gelatinase, an enzyme overexpressed in the $\mathrm{TME}^{148}$. Once the hydrophilic 'head' of the peptide conjugate is removed, the residues assemble into nanofibres mediated by the photosensitizer 'tail'. This assembly-induced retention effect increases the intratumoural half-life of the photosensitizer, enhancing both the photoacoustic imaging signal and the photothermal therapy efficacy. Peptide structures assembled in situ can remain in tumours for up to a month, much longer than intravenously administered peptide nanoparticles ${ }^{143}$. Moreover, post-administration self-assembly offers a way to deliver peptide nanostructures that are unsuitable for intravenous transportation, such as long fibres or gel-like networks. For example, a short peptide-based amphiphile selectively assembles into pericellular fibres in response to hypoxiainduced TME acidity. The fibres formed in situ readily bind to the tumour cell surface and can co-deliver a molecule-targeted drug. In addition, the fibres limit cell motility and contribute to cell death through shape-related lysosomal injury after receptor-mediated internalization, thereby enhancing the therapeutic effect $^{150}$.

In contrast to proteins, bioactive peptides can be synthetically engineered, allowing easy multifunctional design and facile discovery by in vitro and in vivo screening techniques ${ }^{127,151}$. Specific peptide ligands have been developed for a range of targets, including cancer cells, TME cells, extracellular components, tissues susceptible to metastasis and biological barriers ${ }^{143,152,153}$. Furthermore, motifs can be incorporated that enable penetration through lipid bilayers to localize peptide nanoparticles in distinct subcellular compartments, by targeting membranes of the cell, mitochondria or nucleus ${ }^{152,154}$. Importantly, the immunogenicity of peptide assemblies varies considerably with the molecular design. Many peptide assemblies are non-immunogenic in animals, whereas some peptide structures elicit potent antibody responses, which can last for more than a year ${ }^{142,155}$. Therefore, peptides can be designed with different immunogenicity for vaccination or delivery applications.

Therapeutic peptides are a major class of anticancer drugs ${ }^{156}$. However, free peptides suffer from rapid clearance, limited stability in vivo and poor membrane permeability. These limitations can be overcome by fabricating them into nanoparticles. Effector peptides can be released from nanostructures in response to specific stimuli, minimizing the loss of peptide activity owing to the addition of additional moieties, such as targeting motifs. For example, the short peptide T4 (NLLMAAS) is a potent angiogenesis inhibitor, but highly hydrophobic and rapidly cleared from the bloodstream. Using an enzyme-cleavable peptide linker, the sequence can be integrated into $\mathrm{pH}$-responsive self-assembled nanoparticles, which become loose in the weakly acidic TME, leading to the exposure of cleavage sites and eventual release of the small bioactive peptide ${ }^{157}$. Maintaining the nanostructure of apposed peptides at the tumour site may also elicit multivalent or multimeric effects and enhance the potency of therapeutic peptides ${ }^{158}$. For example, in mice, certain immunological effects are elicited only by nanoparticles coated with peptide-major 


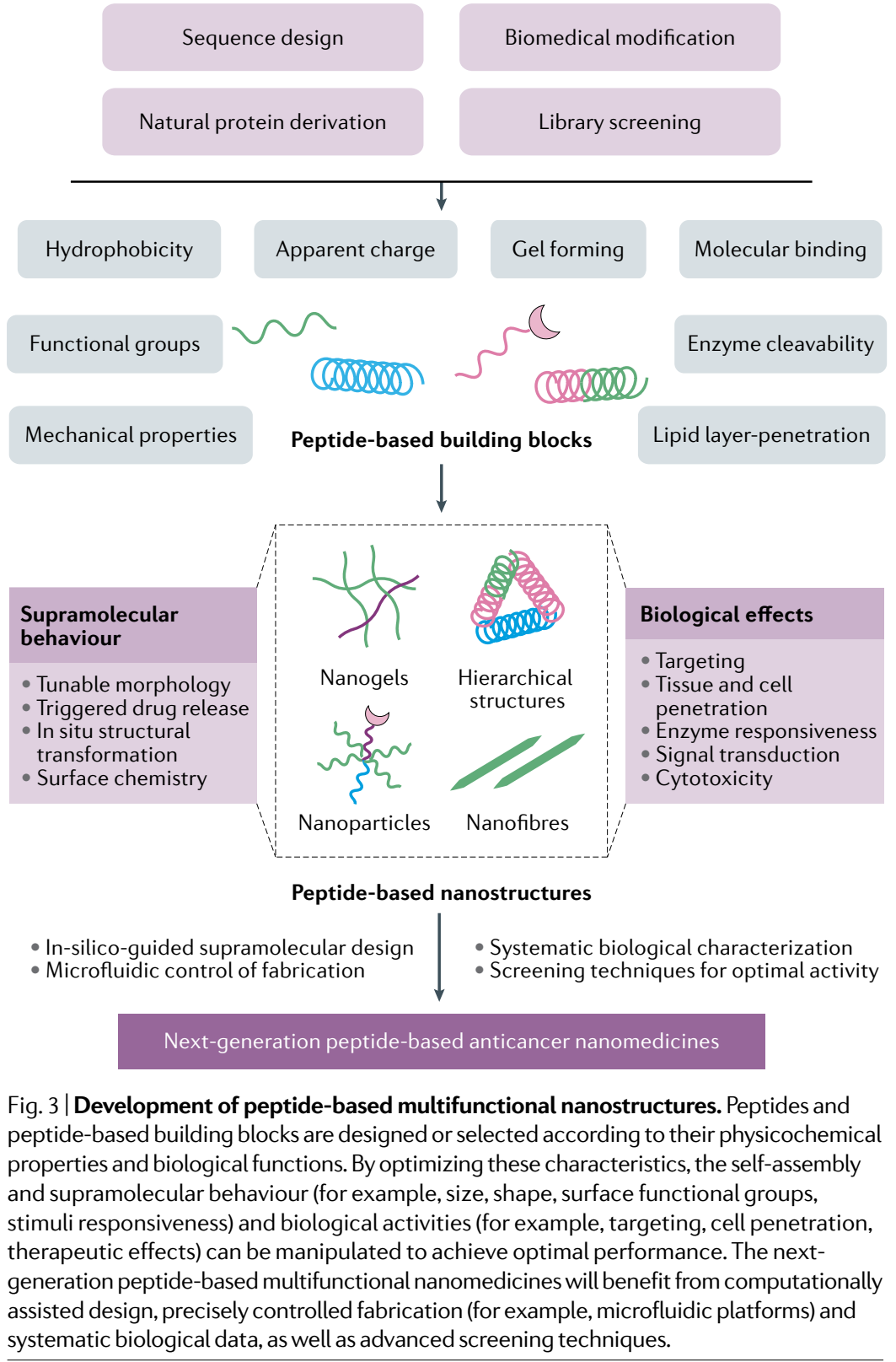

histocompatibility complexes (pMHC) and not by pMHC alone. Here, the high ligand density on the nanoparticle surface accelerates the kinetics of interactions between pMHC and T-cell receptor, and promotes local clustering of receptor molecules on $\mathrm{T}$-cell membranes, amplifying the magnitude of signalling ${ }^{159}$. Although not directly applicable to cancer treatment, this study confirms that a multifunctional nanostructure can elicit greater biological responses than the sum of its individual components, which may be particularly beneficial for peptide and protein nanostructures that can modulate intratumoural signal transduction.

With advanced simulation and fabrication tools, new functional peptides and nanostructures can be created. For example, temperature-sensitive peptide hydrogels have been widely used for long-term drug delivery; however, their function usually depends on complex motifs, making it difficult to further tune their functions on the sequence level. Alternatively, computation-assisted peptide design allows the generation of a library of peptides with accurately tuned thermal hysteresis properties, by alternating the periodical combination of two simple modules ${ }^{160}$. Microfluidics can be used to aid the formation of thermodynamically unfavourable self-assembly products of aromatic dipeptides by maintaining them in a non-equilibrium state, highlighting the possibility of accurately manipulating the formation of peptide assemblies on a kinetic level ${ }^{161}$. Coupled with in vitro and in vivo screening techniques, these methods to precisely predict and control the supramolecular behaviours of peptides will become crucial for the development of multifunctional nanostructures capable of complex actions (FIG. 3).

Natural-protein-derived nanostructures. Proteins have remarkable structural and functional diversity, can be genetically programmed and are involved in essentially all cellular processes. Proteins and their assemblies in particular, caged or virus-like protein structures have long been explored for drug delivery, especially for immunotherapy, owing to their excellent biocompatibility, biodegradability, mechano-chemical features, wide variety of possible modifications, unique molecular recognition and responsive features at precise locations ${ }^{162-165}$. Various nanostructures derived from natural plant and animal proteins (for example, gliadin, legumin, sericin, protamine, collagen, gelatin, serum albumin and ferritin) have been applied for cancer theranostics ${ }^{16-168}$. The preparation method, site-selective modification and recombinant engineering of natural-protein-derived nanostructures, such as emulsion and solvent extraction, desolvation, salt precipitation, polyelectrolyte complexation and electrospraying, are selected based on the physicochemical properties and composition of the proteins and therapeutic agents ${ }^{169}$. For anticancer nanomedicines, the intrinsic biological activities of proteins can be exploited, including drug encapsulation, specific targeting, stimulus-dependent conformational changes, synergistic theranostics and enzymatic activities, epitomizing the multifunctionality of biomolecule-based nanostructures ${ }^{170,171}$. Indeed, nanostructures derived from natural proteins have already found their way into the clinic - for example, albumin nanoparticles ${ }^{172}$.

Serum albumin is the most abundant protein in blood. The long half-life of albumin of 19 days makes it an excellent candidate for drug delivery. Serum albumin naturally crosses vascular endothelia through GP60-receptor-aided transcytosis, and concentrates inside tumours by binding to secreted protein acidic and rich in cysteine (SPARC) ${ }^{173,174}$. Biomimetic penetration of the blood-brain barrier has been achieved using a dual drug-loaded bovine serum albumin nanostructure for glioma targeting and chemotherapy ${ }^{175}$. Proteins possess a variety of functional surface groups, such as thiol, amino and carboxylic groups, that allow drug binding. Indeed, modified serum albumin-based nanostructures have been applied as targeted and responsive cancer nanomedicines in vivo ${ }^{176,177}$. In addition to intravenous administration, an inhalable human serum albumin conjugate can be applied for the treatment 
of drug-resistant lung cancer in a mouse model ${ }^{178}$. A nanovaccine self-assembled in vivo from endogenous human serum albumin and Evans blue-conjugated $\mathrm{CpG}$ or tumour-specific neoantigens has been shown to potentiate the innate and adaptive immunity in multiple syngeneic tumours in mice ${ }^{179}$. Abraxane is the first albumin-based nanomedicine approved by the FDA for the treatment of advanced breast, lung and pancreatic cancer ${ }^{172,180}$. Other albumin-derived anticancer formulations, such as aldoxorubicin, nab-docetaxel and nab-rapamycin, have also been investigated in clinical trials ${ }^{13,181,182}$, highlighting the great promise of albumin-based cancer nanomedicines (TABLE 1).

Ferritin is a ubiquitous, well-characterized protein with a rigid octahedral cage comprising 24 subunits ${ }^{166}$. The cavity of ferritin reversibly self-assembles under acidic conditions, making it an excellent natural nanostructure for therapeutic cargo encapsulation and delivery ${ }^{170}$. The ferritin nanocage binds to transferrin receptor 1, which is overexpressed in a range of cancer cells, particularly in hypoxic tumour tissue ${ }^{183,184}$. Foreign recognition ligands, such as the peptide arginyl-glycylaspartic acid (RDG) and CD47-binding Sirpa variants, introduced by genetic engineering or self-assembling, further expand ferritin functions ${ }^{185,186}$. Ferritin can also function as a bioreactor for the templated synthesis of biomineral nanoparticles, which can be used for cancer imaging and therapy ${ }^{187-190}$. Cancer-targeted catalytic therapy has been explored by engaging the $\mathrm{pH}$-responsive multienzyme-like activities of engineered ferritincarbon nanosphere conjugates ${ }^{191}$. However, the full potential of ferritin-derived nanostructures has yet to be unleashed.

Nanostructures derived from caged proteins are well suited for cancer immunotherapy owing to their geometries and surface properties, which are similar to viruses ${ }^{192-194}$. As vaccine platforms and natural adjuvants, these nanostructures promote passive localization of tumour antigens in the lymphatic system, endocytosis of antigens by APCs and antigen presentation to adaptive immune cells ${ }^{162,163}$. Virus-like nanoparticles (VLPs) are caged protein structures isolated from viruses. VLPs lack infectious genetic material, but feature repetitive surface structures and pathogen-associated molecular patterns, which activate innate immunity and thus can be exploited for cancer vaccine delivery ${ }^{195}$. VLPs from plant viruses (such as cowpea mosaic virus and tobacco mosaic virus) have been thoroughly evaluated for antigen delivery in different cancer models, and vaccine formulations incorporating bacteriophage $\mathrm{Q} \beta$ have shown promise in clinical studies ${ }^{196-200}$.

With their highly ordered structures and symmetry, non-viral protein cages have been engineered to elicit anticancer immune responses ${ }^{201-204}$. For example, by virtue of passive targeting to lymph nodes, antigen-loaded ferritin nanocages can trigger specific cytotoxic CD8 T-cell induction and prolong the survival of colon-tumour-bearing mice ${ }^{185,205}$. Vaccination with tumour antigens caged in heat-shock proteins has proven effective in clinical studies in different cancer types ${ }^{206,207}$. Some cage protein-based adjuvants, such as natural vaults, a ribonucleic protein complex in eukaryotic organisms with a hollow, barrel-shaped structure, favour cell-mediated over humoral immune responses when incorporated into cancer vaccines ${ }^{208}$. Various strategies have been developed for protein-cage-based nanoplatform cancer vaccines to evade neutralization and rapid clearance $^{209}$. However, the exact mechanisms underlying the potential antitumour immunological effects of many natural-protein complexes remain to be explored.

Cells possess many sophisticated functional proteins that perform complex mechanical tasks through stimulus-responsive mechanical motion ${ }^{210}$. The engineering of such proteins into intelligent nanomachines has long been investigated for anticancer therapies, but remains challenging ${ }^{211}$. For example, molecular motor enzymes fuelled by chemical energy show open-closed conformational changes of interlocked movable subunits, triggered by hydrolysis of adenosine- $5^{\prime}$-triphosphate (ATP) ${ }^{212}$. The bacterial chaperonin GroEL has such ATPase activity, and its hydrophobic double-ring cylinder has excellent structural stability as well as heat and acid resistance ${ }^{213}$. These dynamic structural features can be used to release entrapped guest molecules. Indeed, the ATP-switchable cavity of GroEL has been used as smart nanocarrier for the intracellular delivery and release of drugs, membrane proteins and nanoparticles ${ }^{214,215}$. Nanotubes assembled by multiple barrel-shaped GroEL units have been surface functionalized with a boronic acid derivative, which enables cancer cell uptake ${ }^{216}$. Intracellular drug release is triggered through intracellular ATP-responsive disassembly and esterase cleavage. GroEL also binds to plectin1, which is a structural protein highly expressed on some types of cancer cells, and thus can be used to release drugs in response to elevated ATP in the $\mathrm{TME}^{217}$.

Natural-protein-based drug delivery vehicles offer many benefits for cancer therapy; however, clinical translation remains challenging. For example, the structural heterogeneity of proteins leads to chemical and mechanical variability. This problem can be addressed through chemical modification or integration of synthetic materials that do not interfere with the desired in vivo activity ${ }^{7}$. Large-scale synthesis and recombinant protein production at reasonable cost will be required for clinical applications. In addition, formulation stability and long shelf-life are often difficult to achieve owing to the rapid degradation of proteins. Moreover, nanostructure absorption, in vivo distribution, metabolism and excretion must be further clarified by sensitive in vivo detection techniques. The functionalization of nanostructures is restricted by the structures and characteristics of the original proteins. This limitation can be addressed by de novo design and engineering of proteins with tailored therapeutic potential ${ }^{218}$.

\section{Perspectives}

Biomolecule-based nanostructures integrate the material and biological advantages of natural building blocks, enabling the multifunctional design of drug carriers. However, the more complex the materials, the more challenging the clinical translation. In addition, a thorough understanding of nanomaterial-tumour interactions will be required to fully reveal the potential of biomolecule-based anticancer nanostructures. 
Rational design and fabrication. Biomolecule-derived nanomaterials are highly variable in terms of molecular and supramolecular structures, and can interact and interfere with biological pathways in vivo. Therefore, the efficacy of biomolecule-based nanostructures can be particularly sensitive to the molecular composition of their building blocks, which makes system optimization challenging. Computational simulation and statistical models enable the rational design of biomolecule-based nanostructures; for example, molecular dynamics simulations have been applied to screen and identify hydrogel-forming tripeptides ${ }^{219}$. In silico tools can also be used to simulate protein adsorption, extravasation, cell uptake and drug release $^{220,221}$, or to develop structure-activity relationships to analyse safety ${ }^{222}$. However, predicting the in vivo behaviour of biomolecule-based systems is difficult, compared with inorganic or polymeric nanostructures, owing to the complexity of biomolecule-based nanostructures and a lack of systematic experimental in vivo data.

To explore the multifunctional advantage of nanomaterials, systems with highly sophisticated spatial structures and responsive mechanisms have been designed ${ }^{100,101,146}$. However, accurate and reproducible fabrication methods for such complex structures remain limited. Nucleic acids and peptides, whose supramolecular behaviour and thus structure formation can be reproducibly programmed, may provide an advantage, compared with proteins. Microfluidic and high-throughput techniques further allow automation and monitoring of nanomaterial synthesis and characterization ${ }^{161,223,224}$.

Delivery evaluation. A major challenge in the development of tumour-targeted nanomedicines is the lack of tools to estimate and compare in vivo performance. Pharmacokinetic parameters are not always sufficient for describing the mechanism-of-action of nanostructures, such as multimeric and aggregationinduced effects. Current methodologies (for example, chromatography-based small-molecule drug quantification) often cannot discriminate between different nanostructure-associated drug forms, in particular if therapeutic effects are only activated following a multistep mechanism. In addition, the incorporation of active components in biomolecule-based nanostructures often requires complicated strategies. By contrast, most nanoformulations in clinical trials are drug-encapsulating liposomes or polymeric micelles with simple structures, facilitating standardization of in vivo evaluation and comparison with free drug formulations. Therefore, theoretical and statistical models need to be specifically developed for biomolecule-based nanostructures, to pre-clinically assess their action and behaviour in vivo.
Accurate delivery assessment can further be achieved by using probes and contrast agents for high-resolution, real-time in vivo imaging of nanostructures. These probes should ideally differentiate between different nanostructure forms. In particular, owing to differences in the impact of biological barriers on nanostructure transport between individuals, nanomedicines could be coupled with imaging agents (nanotheranostics) to enable real-time delivery evaluation ${ }^{2,12}$. High-content, animal-free evaluation of nanomaterial delivery (for example, using organs-on-a-chip and tumour-on-a-chip devices) may allow nanomedicine screening and drug response prediction ${ }^{24,225}$. However, these models cannot yet accurately predict intratumoural delivery efficiency for individual patients. Machine learning approaches can help to analyse large and heterogeneous datasets and thus may allow the identification of new imaging markers. Several deep learning algorithms based on computed tomography and magnetic resonance imaging have been developed to predict important prognostic factors, such as tumour grade, molecular expression pattern or immune phenotype $\mathrm{e}^{226,227}$. The use of artificial intelligence may enable not only patient stratification, but also the design of personalized nanostructure delivery strategies ${ }^{228}$.

Combating metastasis. Metastasis of tumour cells remains the main cause of cancer-related deaths. Metastasized cancer cells do not necessarily have the same targetable genetic or biological characteristics as primary tumour cells $^{229,230}$. In particular, early metastases are difficult to target by nanoparticle-based strategies owing to the lack of targetable TME characteristics, such as fully developed vasculatures. Nanomedical therapies are being explored for the treatment of metastasis, including early metastases, pre-metastatic tissues and circulating tumour cells ${ }^{1,231}$. For example, nanoparticles coated with biological membranes extracted from pro-metastatic cells, such as platelets ${ }^{232}$ and neutrophils ${ }^{34}$, have been investigated for anti-metastasis drug delivery. Identifying metastatic sites for surgical removal or localized treatments remains difficult, and therefore applying nanostructures that can specifically bind to and target metastatic cancer cells may prove valuable. Multifunctional nanostructures could also incorporate imaging modalities, which is particularly important for the development of anti-metastatic nanomedicines ${ }^{12}$.

Multifunctional nanomedicine is actively explored in preclinical and clinical research. Biomolecule-based nanomaterials are inherently multifunctional, and a thorough understanding of their building blocks, in concert with advances in rational design and in vivo evaluation, will make these nanostructures promising candidates for clinical cancer treatments.

Published online 19 May 2021
1. Shi, J., Kantoff, P. W., Wooster, R. \& Farokhzad, O. C. Cancer nanomedicine: progress, challenges and opportunities. Nat Rev Cancer 17, 20 (2016)

2. Chen, H., Zhang, W., Zhu, G., Xie, J. \& Chen, X. Rethinking cancer nanotheranostics. Nat. Rev. Mater. 2, 17024 (2017).

3. Douglas, S. M., Bachelet, I. \& Church, G. M. A logic-gated nanorobot for targeted transport of molecular payloads. Science 335, 831-834 (2012).

4. Anselmo, A. C. \& Mitragotri, S. Nanoparticles in the clinic: an update. Bioeng. Transl. Med. 4, e10143 (2019).
5. Zhao, Z., Ukidve, A., Kim, J. \& Mitragotri, S. Targeting strategies for tissue-specific drug delivery. Cell 181, 151-167 (2020)

6. Cheng, Z., Al Zaki, A., Hui, J. Z., Muzykantov, V. R. \& Tsourkas, A. Multifunctional nanoparticles: cost versus benefit of adding targeting and imaging capabilities. Science 338, 903 (2012).

7. Datta, L. P., Manchineella, S. \& Govindaraju, T Biomolecules-derived biomaterials. Biomaterials 230, 119633 (2020).

8. Liu, K., Jiang, X. \& Hunziker, P. Carbohydratebased amphiphilic nano delivery systems for cancer therapy. Nanoscale 8, 16091-16156 (2016).

9. Habibi, N., Kamaly, N., Memic, A \& Shafiee, H. Self-assembled peptide-based nanostructures smart nanomaterials toward targeted drug delivery. Nano Today 11, 41-60 (2016).

10. Kakkar, A., Traverso, G., Farokhzad, O. C., Weissleder, R. $\&$ Langer, R. Evolution of macromolecular complexity in drug delivery systems. Nat. Rev. Chem. 1, 63 (2017).

11. Seeman, N. C. \& Sleiman, H. F. DNA nanotechnology. Nat. Rev. Mater. 3, 17068 (2018). 
12. Lammers, T. et al. Cancer nanomedicine: is targeting our target? Nat. Rev. Mater. 1, 16069 (2016).

13. Bobo, D., Robinson, K. J., Islam, J., Thurecht, K. J. \& Corrie, S. R. Nanoparticle-based medicines: a review of FDA-approved materials and clinical trials to date. Pharm. Res. 33, 2373-2387 (2016).

14. Hanahan, D. \& Weinberg, R. A. Hallmarks of cancer: the next generation. Cell 144, 646-674 (2011).

15. Valkenburg, K. C., de Groot, A. E. \& Pienta, K. J. Targeting the tumour stroma to improve cancer therapy. Nat. Rev. Clin. Oncol. 15, 366-381 (2018).

16. Ji, T. et al. Transformable peptide nanocarriers for expeditious drug release and effective cancer therapy via cancer-associated fibroblast activation. Angew. Chem. Int. Ed. 55, 1050-1055 (2016).

17. Han, X. et al. Reversal of pancreatic desmoplasia by re-educating stellate cells with a tumour microenvironment-activated nanosystem. Nat. Commun. 9, 3390 (2018).

18. Li, S. et al. Combination of tumour-infarction therapy and chemotherapy via the co-delivery of doxorubicin and thrombin encapsulated in tumour-targeted nanoparticles. Nat. Biomed. Eng. 4, 732-742 (2020).

19. Metselaar, J. M. \& Lammers, T. Challenges in nanomedicine clinical translation. Drug Deliv. Trans/. Res. 10, 721-725 (2020).

20. Nel, A. E. et al. Understanding biophysicochemical interactions at the nano-bio interface. Nat. Mater. 8, 543-557 (2009)

21. Cai, R. \& Chen, C. The crown and the scepter: roles of the protein corona in nanomedicine. Adv. Mater. 31 1805740 (2019).

22. Banerjee, I., Pangule, R. C. \& Kane, R. S. Antifouling coatings: recent developments in the design of surfaces that prevent fouling by proteins, bacteria and marine organisms. Adv. Mater. 23, 690-718 (2011).

23. Chelmowski, R. et al. Peptide-based SAMs that resist the adsorption of proteins. J. Am. Chem. Soc. 130, 14952-14953 (2008).

24. Tonigold, M. et al. Pre-adsorption of antibodies enables targeting of nanocarriers despite a biomolecular corona. Nat. Nanotechnol. 13, 862-869 (2018).

25. Hamad-Schifferli, K. Exploiting the novel properties of protein coronas: emerging applications in nanomedicine Nanomedicine 10, 1663-1674 (2015).

26. Setten, R. L., Rossi, J. J. \& Han, S.-P. The current state and future directions of RNAi-based therapeutics. Nat. Rev. Drug Discov. 18, 421-446 (2019).

27. Zhang, Z. et al. Brain-targeted drug delivery by manipulating protein corona functions. Nat. Commun 10, 3561 (2019).

28. Mirshafiee, V., Kim, R., Park, S., Mahmoudi, M. \& Kraft, M. L. Impact of protein pre-coating on the protein corona composition and nanoparticle cellular uptake. Biomaterials 75, 295-304 (2016)

29. Owens, D. E. \& Peppas, N. A. Opsonization, biodistribution, and pharmacokinetics of polymeric nanoparticles. Int. J. Pharm. 307, 93-102 (2006)

30. Zhang, P., Sun, F., Liu, S. \& Jiang, S. Anti-PEC antibodies in the clinic: current issues and beyond PEGylation. J. Control. Rel. 244, 184-193 (2016).

31. Hu, C.-M. J. et al. Erythrocyte membrane-camouflaged polymeric nanoparticles as a biomimetic delivery platform. Proc. Natl Acad. Sci. USA 108, 10980 (2011)

32. Rodriguez, P. L. et al. Minimal 'self' peptides that inhibit phagocytic clearance and enhance delivery of nanoparticles. Science 339, 971 (2013).

33. Kim, J. et al. Co-coating of receptor-targeted drug nanocarriers with anti-phagocytic moieties enhances specific tissue uptake versus non-specific phagocytic clearance. Biomaterials 147, 14-25 (2017).

34. Kang, T. et al. Nanoparticles coated with neutrophil membranes can effectively treat cancer metastasis. ACS Nano 11, 1397-1411 (2017).

35. Cheng, Q. et al. Selective organ targeting (SORT) nanoparticles for tissue-specific mRNA delivery and CRISPR-Cas gene editing. Nat. Nanotechnol. 15 313-320 (2020)

36. Blanco, E., Shen, H. \& Ferrari, M. Principles of nanoparticle design for overcoming biological barriers to drug delivery. Nat. Biotechnol. 33, 941 (2015).

37. Nichols, J. W. \& Bae, Y. H. EPR: evidence and fallacy. J. Control. Rel. 190, 451-464 (2014).

38. Nakamura, H., Jun, F. \& Maeda, H. Development of next-generation macromolecular drugs based on the EPR effect: challenges and pitfalls. Expert Opin. Drug Deliv. 12, 53-64 (2015).

39. Wilhelm, S. et al. Analysis of nanoparticle delivery to tumours. Nat. Rev. Mater. 1, 16014 (2016).

40. Li, S. et al. Nanoparticle-mediated local depletion of tumour-associated platelets disrupts vascular barriers and augments drug accumulation in tumours. Nat. Biomed. Eng. 1, 667-679 (2017).

41. Miller, M. A. et al. Predicting therapeutic nanomedicine efficacy using a companion magnetic resonance imaging nanoparticle. Sci. Transl. Med. 7 314 ra183 (2015).

42. Shah, S., Liu, Y., Hu, W. \& Gao, J. Modeling particle shape-dependent dynamics in nanomedicine. J. Nanosci. Nanotechnol. 11, 919-928 (2011).

43. Liang, Q. et al. The softness of tumour-cell-derived microparticles regulates their drug-delivery efficiency. Nat. Biomed. Eng. 3, 729-740 (2019).

44. Palomba, R. et al. Biomimetic carriers mimicking leukocyte plasma membrane to increase tumor vasculature permeability. Sci. Rep. 6, 34422 (2016).

45. Sindhwani, S. et al. The entry of nanoparticles into solid tumours. Nat. Mater 19, 566-575 (2020).

46. Liu, X., Jiang, J. \& Meng, H. Transcytosis - an effective targeting strategy that is complementary to 'EPR effect' for pancreatic cancer nano drug delivery. Theranostics 9, 8018-8025 (2019).

47. Pandit, S., Dutta, D. \& Nie, S. Active transcytosis and new opportunities for cancer nanomedicine. Nat. Mater. 19, 478-480 (2020).

48. Guo, Y. et al. Erythrocyte membrane-enveloped polymeric nanoparticles as nanovaccine for induction of antitumor immunity against melanoma. ACS Nano 9, 6918-6933 (2015).

49. Xu, J. et al. Reversing tumor stemness via orally targeted nanoparticles achieves efficient colon cancer treatment. Biomaterials 216, 119247 (2019).

50. Shields, C. W. et al. Cellular backpacks for macrophage immunotherapy. Sci. Adv. 6, eaaz6579 (2020).

51. Ngambenjawong, C., Gustafson, H. H. \& Pun, S. H. Progress in tumor-associated macrophage (TAM)targeted therapeutics. Adv. Drug Deliv. Rev. 114 206-221 (2017)

52. Dowdy, S. F. Overcoming cellular barriers for RNA therapeutics. Nat. Biotechnol. 35, 222-229 (2017)

53. Thambi, T., Phan, V. H. G. \& Lee, D. S. Stimuli-sensitive injectable hydrogels based on polysaccharides and their biomedical applications. Macromol. Rapid Commun. 37, 1881-1896 (2016)

54. Lin, N., Huang, J. \& Dufresne, A. Preparation, properties and applications of polysaccharide nanocrystals in advanced functional nanomaterials: a review. Nanoscale 4, 3274-3294 (2012).

55. $\mathrm{Hu}, \mathrm{H}$. et al. Redox-responsive polycationfunctionalized cotton cellulose nanocrystals for effective cancer treatment. ACS Appl. Mater. Interfaces 7, 8942-8951 (2015).

56. Swierczewska, M. Han, H. S., Kim, K., Park, J. H. \& Lee, S. Polysaccharide-based nanoparticles for theranostic nanomedicine. Adv. Drug Deliv. Rev. 99 70-84 (2016)

57. Butt, A. M. et al. Doxorubicin and siRNA codelivery via chitosan-coated $\mathrm{pH}$-responsive mixed micellar polyplexes for enhanced cancer therapy in multidrug-resistant tumors. Mol. Pharm. 13 4179-4190 (2016).

58. Shi, G.-N. et al. Enhanced antitumor immunity by targeting dendritic cells with tumor cell lysate-loaded chitosan nanoparticles vaccine. Biomaterials 113 191-202 (2017)

59. Thambi, T. et al. Bioreducible carboxymethyl dextran nanoparticles for tumor-targeted drug delivery. Adv. Healthc. Mater. 3, 1829-1838 (2014).

60. Han, H. S. et al. Bioreducible shell-cross-linked hyaluronic acid nanoparticles for tumor-targeted drug delivery. Biomacromolecules 16, 447-456 (2015).

61. Wang, X. et al. Amphiphilic carboxymethyl chitosanquercetin conjugate with P-gp inhibitory properties for oral delivery of paclitaxel. Biomaterials 35 7654-7665 (2014).

62. Schmidt, K. T. et al. Measurement of NLG207 (formerly CRLX101) nanoparticle-bound and released camptothecin in human plasma. J. Pharm. Biomed. Anal. 181, 113073 (2020).

63. Choi, K. Y. et al. Versatile RNA interference nanoplatform for systemic delivery of RNAs. ACS Nano 8, 4559-4570 (2014).

64. Lallana, E. et al. Chitosan/hyaluronic acid nanoparticles: rational design revisited for RNA delivery. Mol. Pharm. 14, 2422-2436 (2017).

65. Kim, T. et al. Nanoparticle-patterned multicompartmental chitosan capsules for oral delivery of oligonucleotides. ACS Biomater. Sci. Eng. 4 4163-4173 (2018).

66. Wu, L. et al. A review about the development of fucoidan in antitumor activity: progress and challenges. Carbohydr. Polym. 154, 96-111 (2016).
67. Shamay, Y. et al. P-selectin is a nanotherapeutic delivery target in the tumor microenvironment. Sci. Transl. Med. 8, 345ra87 (2016).

68. Juenet, M. et al. Thrombolytic therapy based on fucoidan-functionalized polymer nanoparticles targeting P-selectin. Biomaterials 156, 204-216 (2018).

69. Otterlei, M. et al. Induction of cytokine production from human monocytes stimulated with alginate. J. Immunother. 10, 286-291 (1991)

70. Jani, M. S., Veetil, A. T. \& Krishnan, Y. Precision immunomodulation with synthetic nucleic acid technologies. Nat. Rev. Mater. 4, 451-458 (2019).

71. Zhao, Y. et al. Nanofabrication based on DNA nanotechnology. Nano Today 26, 123-148 (2019).

72. Seeman, N. C. Nucleic acid junctions and lattices. J. Theor. Biol. 99, 237-247 (1982).

73. Guo, P., Zhang, C., Chen, C., Garver, K. \& Trottier, M. Inter-RNA interaction of phage phi29 pRNA to form a hexameric complex for viral DNA transportation. Mol. Cell 2, 149-155 (1998).

74. Guo, P. The emerging field of RNA nanotechnology. Nat. Nanotechnol. 5, 833-842 (2010).

75. Xu, C. et al. Favorable biodistribution, specific targeting and conditional endosomal escape of RNA nanoparticles in cancer therapy. Cancer Lett. 414, 57-70 (2018)

76. Jasinski, D., Haque, F., Binzel, D. W. \& Guo, P. Advancement of the emerging field of RNA nanotechnology. ACS Nano 11, 1142-1164 (2017).

77. Watts, J. K., Deleavey, G. F. \& Damha, M. J. Chemically modified siRNA: tools and applications. Drug Discov. Today 13, 842-855 (2008).

78. Piao, X., Xia, X., Mao, J. \& Bong, D. Peptide ligation and RNA cleavage via an abiotic template interface. J. Am. Chem. Soc. 137, 3751-3754 (2015).

79. Winfree, E., Liu, F., Wenzler, L. A. \& Seeman, N. C. Design and self-assembly of two-dimensional DNA crystals. Nature 394, 539-544 (1998).

80. Rothemund, P. W. Folding DNA to create nanoscale shapes and patterns. Nature 440, 297-302 (2006).

81. Wei, B., Dai, M. \& Yin, P. Complex shapes self-assembled from single-stranded DNA tiles. Nature 485, 623-626 (2012).

82. Ali, M. M. et al. Rolling circle amplification: a versatile tool for chemical biology, materials science and medicine. Chem. Soc. Rev. 43, 3324-3341 (2014).

83. Wang, C. Sun, W. Wright, G., Wang, A. Z. \& Gu, Z Inflammation-triggered cancer immunotherapy by programmed delivery of $\mathrm{CpG}$ and anti-PD 1 antibody. Adv. Mater. 28, 8912-8920 (2016).

84. Ramezani, H. \& Dietz, H. Building machines with DNA molecules. Nat. Rev. Genet. 21, 5-26 (2020).

85. Wang, P., Ko, S. H., Tian, C., Hao, C. \& Mao, C. RNA-DNA hybrid origami: folding of a long RNA single strand into complex nanostructures using short DNA helper strands. Chem. Commun. 49 5462-5464 (2013).

86. Zhou, J. \& Rossi, J. Aptamers as targeted therapeutics: current potential and challenges. Nat. Rev. Drug Discov. 16, 181-202 (2017).

87. Li, L. et al. Nucleic acid aptamers for molecular diagnostics and therapeutics: advances and perspectives. Angew. Chem. Int. Ed. https://doi.org/ 10.1002/anie.202003563 (2020).

88. Ren, K. et al. A DNA dual lock-and-key strategy for cell-subtype-specific siRNA delivery. Nat. Commun. 7 , 13580 (2016)

89. Ma, W. et al. An intelligent DNA nanorobot with in vitro enhanced protein lysosomal degradation of HER2. Nano Lett. 19, 4505-4517 (2019).

90. Jiang, D. et al. Multiple-armed tetrahedral DNA nanostructures for tumor-targeting, dual-modality in vivo imaging. ACS Appl. Mater. Interfaces 8 4378-4384 (2016)

91. Lee, H. et al. Molecularly self-assembled nucleic acid nanoparticles for targeted in vivo siRNA delivery. Nat. Nanotechnol. 7, 389-393 (2012).

92. Zhang, Q. et al. DNA origami as an in vivo drug delivery vehicle for cancer therapy. ACS Nano $\mathbf{8}$, 6633-6643 (2014)

93. Yang, Y. et al. G-quadruplex-based nanoscale coordination polymers to modulate tumor hypoxia and achieve nuclear-targeted drug delivery for enhanced photodynamic therapy. Nano Lett. 18 6867-6875 (2018).

94. Veetil, A. T. et al. Cell-targetable DNA nanocapsules for spatiotemporal release of caged bioactive small molecules. Nat. Nanotechnol. 12, 1183-1189 (2017).

95. Bujold, K. E., Hsu, J. C. C. \& Sleiman, H. F. Optimized DNA 'nanosuitcases' for encapsulation and conditional release of siRNA. J. Am. Chem. Soc. 138 14030-14038 (2016). 
96. Ranallo, S., Prevost-Tremblay, C., Idili, A Vallee-Belisle, A. \& Ricci, F. Antibody-powered nucleic acid release using a DNA-based nanomachine. Nat. Commun. 8, 15150 (2017).

97. Amir, Y. et al. Universal computing by DNA origami robots in a living animal. Nat. Nanotechnol. 9, 353-357 (2014)

98. Arnon, S. et al. Thought-controlled nanoscale robots in a living host. PLOS ONE 11, e0161227 (2016).

99. Li, S. et al. A DNA nanorobot functions as a cancer therapeutic in response to a molecular trigger in vivo. Nat. Biotechnol. 36, 258-264 (2018).

100. Ma, P. Q., Huang, Q., Li, H. D., Yin, B. C. \& Ye, B. C. Multimachine communication network that mimics the adaptive immune response. J. Am. Chem. Soc. 142 3851-3861 (2020)

101. Kobori, S., Nomura, Y. \& Yokobayashi, Y. Self-powered RNA nanomachine driven by metastable structure. Nucleic Acids Res. 47, 6007-6014 (2019).

102. Agrahari, V. et al. Intelligent micro-/nanorobots as drug and cell carrier devices for biomedical therapeutic advancement: promising development opportunities and translational challenges. Biomaterials 260, 120163 (2020)

103. Wu, Z., Chen, Y., Mukasa, D., Pak, O. S. \& Gao, W. Medical micro/nanorobots in complex media. Chem. Soc. Rev. 49, 8088-8112 (2020).

104. Hong, E. et al. Structure and composition define immunorecognition of nucleic acid nanoparticles. Nano Lett. 18, 4309-4321 (2018).

105. Pardi, N., Hogan, M. J., Porter, F. W. \& Weissman, D. mRNA vaccines - a new era in vaccinology. Nat. Rev. Drug Discov. 17, 261-279 (2018).

106. Jackson, N. A. C., Kester, K. E., Casimiro, D., Gurunathan, S. \& DeRosa, F. The promise of mRNA vaccines: a biotech and industrial perspective. NPJ Vaccines 5, 11 (2020)

107. Jeyanathan, M. et al. Immunological considerations for COVID-19 vaccine strategies. Nat. Rev. Immunol 20, 615-632 (2020)

108. Keller, A. \& Linko, V. Challenges and perspectives of DNA nanostructures in biomedicine. Angew. Chem. Int. Ed. 59, 15818-15833 (2020).

109. Afonin, K. A. et al. Triggering of RNA interference with RNA-RNA, RNA-DNA, and DNA-RNA nanoparticles. ACS Nano 9, 251-259 (2015).

110. Guo, S. et al. Size, shape, and sequence-dependent immunogenicity of RNA nanoparticles. Mol. Ther Nucleic Acids 9, 399-408 (2017).

111. Halman, J. R. et al. Functionally-interdependent shape-switching nanoparticles with controllable properties. Nucleic Acids Res. 45, 2210-2220 (2017).

112. Guo, S. et al. Ultra-thermostable RNA nanoparticles for solubilizing and high-yield loading of paclitaxe for breast cancer therapy. Nat. Commun. 11, 972 (2020).

113. Hyde, J. L. et al. A viral RNA structural element alters host recognition of nonself RNA. Science 343 783-787 (2014)

114. Rackley, L. et al. RNA fibers as optimized nanoscaffolds for siRNA coordination and reduced immunological recognition. Adv. Funct. Mater. 28, 1805959 (2018)

115. Liu, J. et al. A tailored DNA nanoplatform for synergistic RNAi-/chemotherapy of multidrug-resistant tumors. Angew. Chem. Int. Ed. 57, 15486-15490 (2018).

116. Surana, S., Shenoy, A. R. \& Krishnan, Y. Designing DNA nanodevices for compatibility with the immune system of higher organisms. Nat. Nanotechnol. 10, 741-747 (2015)

117. Johnson, M. B. et al. Programmable nucleic acid based polygons with controlled neuroimmunomodulatory properties for predictive QSAR modeling. Small 13, 1701255 (2017)

118. Yamankurt, G. et al. Exploration of the nanomedicinedesign space with high-throughput screening and machine learning. Nat. Biomed. Eng. 3, 318-327 (2019).

119. Kroll, A. V. et al. Biomimetic nanoparticle vaccines for cancer therapy. Adv. Biosyst. 3, 1800219 (2019).

120. Klinman, D. M. Immunotherapeutic uses of $\mathrm{CpC}$ oligodeoxynucleotides. Nat. Rev. Immunol. 4 , 249-258 (2004)

121. Khisamutdinov, E. F. et al. Enhancing immunomodulation on innate immunity by shape transition among RNA triangle, square and pentagon nanovehicles. Nucleic Acids Res. 42, 9996-10004 (2014)

122. Liu, X. et al. A DNA nanostructure platform for directed assembly of synthetic vaccines. Nano Lett. 12, 4254-4259 (2012).
123. Li, J. et al. Self-assembled multivalent DNA nanostructures for noninvasive intracellular delivery of immunostimulatory $\mathrm{CpG}$ oligonucleotides. ACS Nano 5, 8783-8789 (2011).

124. Goldberg, M. S. Improving cancer immunotherapy through nanotechnology. Nat. Rev. Cancer 19, 587-602 (2019)

125. Wang, S. et al. Rational vaccinology with spherical nucleic acids. Proc. Natl Acad. Sci. USA 116 10473-10481 (2019).

126. Umeki, Y. et al. Induction of ptent antitumor immunity by sustained release of cationic antigen from a DNA-based hydrogel with adjuvant activity. Adv. Funct. Mater. 25, 5758-5767 (2015).

127. Zhu, G. et al. Intertwining DNA-RNA nanocapsules loaded with tumor neoantigens as synergistic nanovaccines for cancer immunotherapy. Nat. Commun. 8, 1482 (2017).

128. Santulli-Marotto, S., Nair, S. K., Rusconi, C., Sullenger, B. \& Gilboa, E. Multivalent RNA aptamers that inhibit CTLA-4 and enhance tumor immunity. Cancer Res. 63, 7483-7489 (2003).

129. Devaraj, S. G. T., Rao, L. G. L., Zu, Y., Chang, J. \& lyer, S. P. DNA aptamer against anti-programmed cell death-1 (anti-PD1-apt) induces robust anti-leukemic activity in vitro and in vivo humanized NSG mice with myeloid leukemia xenografts. Blood 130, 1373 (2017).

130. Praetorius, F. et al. Biotechnological mass production of DNA origami. Nature 552, 84-87 (2017)

131. Li, M. et al. In vivo production of RNA nanostructures via programmed folding of single-stranded RNAs. Nat. Commun. 9, 2196 (2018)

132. Bae, W., Kocabey, S. \& Liedl, T. DNA nanostructures in vitro, in vivo and on membranes. Nano Today 26 98-107 (2019).

133. Gerling, T., Kube, M., Kick, B. \& Dietz, H. Sequenceprogrammable covalent bonding of designed DNA assemblies. Sci. Adv. 4, eaau 1157 (2018)

134. Anastassacos, F. M., Zhao, Z., Zeng, Y. \& Shih, W. M Glutaraldehyde cross-linking of oligolysines coating DNA origami greatly reduces susceptibility to nuclease degradation. J. Am. Chem. Soc. 142, 3311-3315 (2020)

135. Kim, Y. \& Yin, P. Enhancing biocompatible stability of DNA nanostructures using dendritic oligonucleotides and brick motifs. Angew. Chem. Int. Ed. 59, 700-703 (2020).

136. Perrault, S. D. \& Shih, W. M. Lipid membrane encapsulation of a 3D DNA nano octahedron. Methods Mol. Biol. 1500, 165-184 (2017).

137. Wiraja, C. et al. Framework nucleic acids as programmable carrier for transdermal drug delivery. Nat. Commun. 10, 1147 (2019).

138. Benson, E. et al. DNA rendering of polyhedral meshes at the nanoscale. Nature 523, 441-444 (2015).

139. Veneziano, R. et al. Designer nanoscale DNA assemblies programmed from the top down. Science 352, 1534 (2016)

140. De Santis, E. \& Ryadnov, M. G. Peptide self-assembly for nanomaterials: the old new kid on the block. Chem. Soc. Rev. 44, 8288-8300 (2015).

141. Qin, H., Ding, Y., Mujeeb, A., Zhao, Y. \& Nie, C. Tumor microenvironment targeting and responsive peptide-based nanoformulations for improved tumor therapy. Mol. Pharm. 92, 219-231 (2017).

142. Eskandari, S., Guerin, T., Toth, I. \& Stephenson, R. J. Recent advances in self-assembled peptides: implications for targeted drug delivery and vaccine engineering. Adv. Drug Deliv. Rev. 110-111, 169-187 (2017).

143. Qi, G.-B., Gao, Y.-J., Wang, L. \& Wang, H. Self-assembled peptide-based nanomaterials for biomedical imaging and therapy. Adv. Mater. 30 , 1703444 (2018)

144. Liang, C. et al. Enzyme-assisted peptide folding assembly and anti-cancer properties. Nanoscale $\mathbf{9}$, 11987-11993 (2017).

145. Beesley, J. L. et al. Modifying self-assembled peptide cages to control internalization into mammalian cells. Nano Lett. 18, 5933-5937 (2018)

146. Ueda, M. et al. End-sealed high aspect ratio hollow nanotubes encapsulating an anticancer drug: torpedo-shaped peptidic nanocapsules. ACS Nano 13 305-312 (2019)

147. Wyatt, L. C. et al. Peptides of pHLIP family for targeted intracellular and extracellular delivery of cargo molecules to tumors. Proc. Natl Acad. Sci. USA 115, E2811-E2818 (2018)

148. Zhang, D. et al. In situ formation of nanofibers from purpurin 18-peptide conjugates and the assembly induced retention effect in tumor sites. Adv. Mater. 27, 6125-6130 (2015)
149. Dimatteo, R., Darling, N. J. \& Segura, T. In situ forming injectable hydrogels for drug delivery and wound repair. Adv. Mater. 127, 167-184 (2018)

150. Li, J. et al. New power of self-assembling carbonic anhydrase inhibitor: short peptide-constructed nanofibers inspire hypoxic cancer therapy. Sci. Adv. 5 , eaax0937 (2019).

151. Mann, A. P. et al. A peptide for targeted, systemic delivery of imaging and therapeutic compounds into acute brain injuries. Nat. Commun. 7, 11980 (2016).

152. Zhang, C. et al. Peptide-based multifunctional nanomaterials for tumor imaging and therapy. Adv. Funct. Mater. 28, 1804492 (2018).

153. Zhang, G. et al. A delivery system targeting bone formation surfaces to facilitate RNAi-based anabolic therapy. Nat. Med. 18, 307-314 (2012).

154. Field, L. D., Delehanty, J. B., Chen, Y. \& Medintz, I. L. Peptides for specifically targeting nanoparticles to cellular organelles: quo vadis? Acc. Chem. Res. 48 1380-1390 (2015).

155. Rudra, J. S. et al. Modulating adaptive immune responses to peptide self-assemblies. ACS Nano 6 , 1557-1564 (2012).

156. Lau, J. L. \& Dunn, M. K. Therapeutic peptides: historical perspectives, current development trends, and future directions. Bioorg. Med. Chem. 26, 2700-2707 (2018).

157. Zhang, L. et al. Cooperatively responsive peptide nanotherapeutic that regulates angiopoietin receptor Tie2 activity in tumor microenvironment to prevent breast tumor relapse after chemotherapy. ACS Nano 13, 5091-5102 (2019)

158. Gray, B. P., Li, S. \& Brown, K. C. From phage display to nanoparticle delivery: functionalizing liposomes with multivalent peptides improves targeting to a cancer biomarker. Bioconjug. Chem. 24, 85-96 (2013).

159. Singha, S. et al. Peptide-MHC-based nanomedicines for autoimmunity function as T-cell receptor microclustering devices. Nat. Nanotechnol. 12 701-710 (2017)

160. Roberts, S. et al. Injectable tissue integrating networks from recombinant polypeptides with tunable order. Nat. Mater. 17, 1154-1163 (2018).

161. Mason, T. O. et al. Synthesis of nonequilibrium supramolecular peptide polymers on a microfluidic platform. J. Am. Chem. Soc. 138, 9589-9596 (2016).

162. Molino, N. M. \& Wang, S. W. Caged protein nanoparticles for drug delivery. Curr. Opin. Biotechnol. 28, 75-82 (2014).

163. Neek, M., Kim, T. I. \& Wang, S.-W. Protein-based nanoparticles in cancer vaccine development. Nanomedicine 15, 164-174 (2019).

164. Zhuang, J. et al. Nanoparticle delivery of immunostimulatory agents for cancer immunotherapy. Theranostics 9, 7826-7848 (2019).

165. Edwardson, T. G. W. \& Hilvert, D. Virus-inspired function in engineered protein cages. J. Am. Chem. Soc. 141, 9432-9443 (2019)

166. Cannon, K. A., Ochoa, J. M. \& Yeates, T. O. High-symmetry protein assemblies: patterns and emerging applications. Curr. Opin. Struct. Biol. $\mathbf{5 5}$ 77-84 (2019).

167. Jain, A., Singh, S. K., Arya, S. K., Kundu, S. C. \& Kapoor, S. Protein nanoparticles: promising platforms for drug delivery applications. ACS Biomater. Sci. Eng. 4, 3939-3961 (2018)

168. Yassine, H. M. et al. Hemagglutinin-stem nanoparticles generate heterosubtypic influenza protection. Nat. Med. 21, 1065-1070 (2015).

169. Kuan, S. L, Bergamini, F. R. G \& Weil, T. Functiona protein nanostructures: a chemical toolbox. Chem. Soc. Rev. 47, 9069-9105 (2018).

170. He, J., Fan, K. \& Yan, X. Ferritin drug carrier (FDC) for tumor targeting therapy. J. Control. Rel. 311 , 288-300 (2019)

171. Jiang, B., Fang, L., Wu, K., Yan, X. \& Fan, K. Ferritins as natural and artificial nanozymes for theranostics. Theranostics 10, 687-706 (2020)

172. Kratz, F. A clinical update of using albumin as a drug vehicle - a commentary. J. Control. Rel. 190 331-336 (2014).

173. Desai, N., Trieu, V., Damascelli, B. \& Soon-Shiong, P. SPARC expression correlates with tumor response to albumin-bound paclitaxel in head and neck cancer patients. Transl. Oncol. 2, 59-64 (2009).

174. Hidalgo, M. et al. SPARC expression did not predict efficacy of nab-paclitaxel plus gemcitabine or gemcitabine alone for metastatic pancreatic cancer in an exploratory analysis of the phase III MPACT trial. Clin. Cancer Res. 21, 4811-4818 (2015). 
175. Lin, T. et al. Blood-brain-barrier-penetrating albumin nanoparticles for biomimetic drug delivery via albumin-binding protein pathways for antiglioma therapy. ACS Nano 10, 9999-10012 (2016).

176. Chung, S. W. et al. Albumin-binding caspase-cleavable prodrug that is selectively activated in radiation exposed local tumor. Biomaterials 94, 1-8 (2016).

177. Sarett, S. M. et al. Lipophilic siRNA targets albumin in situ and promotes bioavailability, tumor penetration, and carrier-free gene silencing. Proc. Nat Acad. Sci. USA 114, E6490-E6497 (2017).

178. Choi, S. H. et al. Inhalable self-assembled albumin nanoparticles for treating drug-resistant lung cancer. J. Control. Rel. 197, 199-207 (2015).

179. Zhu, G. et al. Albumin/vaccine nanocomplexes that assemble in vivo for combination cancer immunotherapy. Nat. Commun. 8, 1954 (2017).

180. Gradishar, W. J. Albumin-bound paclitaxel: a next-generation taxane. Expert Opin. Pharmacother. 7, 1041-1053 (2006)

181. Chawla, S. P. et al. First-line aldoxorubicin vs doxorubicin in metastatic or locally advanced unresectable soft-tissue sarcoma: a phase $2 \mathrm{~b}$ randomized clinical trial. JAMA Oncol. 1, 1272-1280 (2015).

182. Cirstea, D. et al. Dual inhibition of akt/mammalian target of rapamycin pathway by nanoparticle albumin-bound-rapamycin and perifosine induces antitumor activity in multiple myeloma. Mol. Cancer Ther. 9, 963-975 (2010).

183. Huang, X. L. et al. Hypoxia-tropic protein nanocages for modulation of tumor- and chemotherapy-associated hypoxia. ACS Nano 13, 236-247 (2019).

184. Fan, K. et al. Ferritin nanocarrier traverses the blood brain barrier and kills glioma. ACS Nano 12 , 4105-4115 (2018)

185. Lee, E. J. et al. Nanocage-therapeutics prevailing phagocytosis and immunogenic cell death awakens immunity against cancer. Adv. Mater. 30, 11 (2018).

186. Zhen, Z. et al. RGD-modified apoferritin nanoparticles for efficient drug delivery to tumors. ACS Nano 7 , 4830-4837 (2013)

187. Wang, Z. et al. Biomineralization-inspired synthesis of copper sulfide-ferritin nanocages as cancer theranostics. ACS Nano 10, 3453-3460 (2016).

188. Sun, C. et al. Controlling assembly of paired gold clusters within apoferritin nanoreactor for in vivo kidney targeting and biomedical imaging. J. Am. Chem. Soc. 133, 8617-8624 (2011).

189. Fan, J. et al. Direct evidence for catalase and peroxidase activities of ferritin-platinum nanoparticles. Biomaterials 32, 1611-1618 (2011)

190. Sun, C. et al. Fine-tuned h-ferritin nanocage with multiple gold clusters as near-infrared kidney specific targeting nanoprobe. Bioconjug. Chem. 26, 193-196 (2015).

191. Fan, K. et al. In vivo guiding nitrogen-doped carbon nanozyme for tumor catalytic therapy. Nat. Commun 9, 1440 (2018)

192. Shan, W. J. et al. Bioengineered nanocage from $\mathrm{HBc}$ protein for combination cancer immunotherapy. Nano Lett 19, 1719-1727 (2019).

193. Chowdhury, S. et al. Programmable bacteria induce durable tumor regression and systemic antitumor immunity. Nat. Med. 25, 1057-1063 (2019).

194. Tang, L. et al. Enhancing T cell therapy through TCR-signaling-responsive nanoparticle drug delivery. Nat. Biotechnol. 36, 707-716 (2018).

195. Mohsen, M. O., Zha, L., Cabral-Miranda, G. \& Bachmann, M. F. Major findings and recent advances in virus-like particle (VLP)-based vaccines. Semin. Immunol. 34, 123-132 (2017).

196. Shukla, S. et al. Plant viral nanoparticles-based HER2 vaccine: immune response influenced by differential transport, localization and cellular interactions of particulate carriers. Biomaterials 121, 15-27 (2017).

197. Lizotte, P. H. et al. In situ vaccination with cowpea mosaic virus nanoparticles suppresses metastatic cancer. Nat. Nanotechnol. 11, 295-303 (2016).

198. Kemnade, J. O. et al. Tobacco mosaic virus efficiently targets DC uptake, activation and antigen-specific $\mathrm{T}$ cell responses in vivo. Vaccine $32,4228-4233$ (2014).

199. Goldinger, S. M. et al. Nano-particle vaccination combined with TLR-7 and -9 ligands triggers memory and effector CD8(+) T-cell responses in melanoma patients. Eur. J. Immunol. 42, 3049-3061 (2012)

200. Serradell, M. C. et al. Efficient oral vaccination by bioengineering virus-like particles with protozoan surface proteins. Nat. Commun. 10, 361 (2019).
201. Molino, N. M., Neek, M., Tucker, J. A., Nelson, E. L. \& Wang, S. W. Viral-mimicking protein nanoparticle vaccine for eliciting anti-tumor responses. Biomaterials 86, 83-91 (2016).

202. Neek, M. et al. Co-delivery of human cancer-testis antigens with adjuvant in protein nanoparticles induces higher cell-mediated immune responses. Biomaterials 156, 194-203 (2018).

203. Molino, N. M., Neek, M., Tucker, J. A., Nelson, E. L. \& Wang, S. W. Display of DNA on nanoparticles for targeting antigen presenting cells. ACS Biomater. Sci. Eng. 3, 496-501 (2017).

204. Molino, N. M., Anderson, A. K., Nelson, E. L. \& Wang, S. W. Biomimetic protein nanoparticles facilitate enhanced dendritic cell activation and cross-presentation. ACS Nano 7, 9743-9752 (2013).

205. Han, J. A et al. Ferritin protein cage nanoparticles as versatile antigen delivery nanoplatforms for dendritic cell (DC)-based vaccine development. Nanomedicine 10, 561-569 (2014).

206. Specht, H. M. et al. Heat shock protein 70 (Hsp70) peptide activated natural killer (NK) cells for the treatment of patients with non-small cell lung cancer (NSCLC) after radiochemotherapy (RCTx) - from preclinical studies to a clinical phase II trial. Front Immunol. 6, 162 (2015).

207. Crane, C. A. et al. Individual patient-specific immunity against high-grade glioma after vaccination with autologous tumor derived peptides bound to the 96 KD chaperone protein. Clin. Cancer Res. 19 , 205-214 (2013).

208. Kar, U. K. et al. Vault nanocapsules as adjuvants favor cell-mediated over antibody-mediated immune responses following immunization of mice. PLOS ONE 7, e38553 (2012).

209. Schwarz, B. et al. Symmetry controlled, genetic presentation of bioactive proteins on the $\mathrm{P} 22$ virus-like particle using an external decoration protein. ACS Nano 9, 9134-9147 (2015)

210. van den Heuvel, M. G. L. \& Dekker, C. Motor proteins at work for nanotechnology. Science 317, 333-336 (2007).

211. Chen, H. B. et al. Precise nanomedicine for intelligent therapy of cancer. Sci. China Chem. 61, 1503-1552 (2018).

212. Kinbara, K. \& Aida, T. Toward intelligent molecular machines: directed motions of biological and artificial molecules and assemblies. Chem. Rev. 105 1377-1400 (2005).

213. Hayer-Hartl, M., Bracher, A. \& Hartl, F. U. The GroEL-GroES chaperonin machine: a nano-cage for protein folding. Trends Biochem. Sci. 41, 62-76 (2016).

214. Ishii, D. et al. Chaperonin-mediated stabilization and ATP-triggered release of semiconductor nanoparticles. Nature 423, 628-632 (2003)

215. Deaton, J. et al. Functional bacteriorhodopsin is efficiently solubilized and delivered to membranes by the chaperonin GroEL. Proc. Natl Acad. Sci. USA 101 2281-2286 (2004).

216. Biswas, S. et al. Biomolecular robotics for chemomechanically driven guest delivery fuelled by intracellular ATP. Nat. Chem. 5, 613-620 (2013).

217. Yuan, Y. et al. Chaperonin-GroEL as a smart hydrophobic drug delivery and tumor targeting molecular machine for tumor therapy. Nano Lett. 18, 921-928 (2018)

218. Kuhlman, B. \& Bradley, P. Advances in protein structure prediction and design. Nat. Rev. Mol. Cell Biol. 20, 681-697 (2019)

219. Frederix, P. W. J. M. et al. Exploring the sequence space for (tri-)peptide self-assembly to design and discover new hydrogels. Nat. Chem. 7, 30-37 (2015).

220. Sacha, G. M. \& Varona, P. Artificial intelligence in nanotechnology. Nanotechnology 24, 452002 (2013).

221. Pietro, M. \& Bernhard Aribo, S. In silico models for nanomedicine: recent developments. Curr. Med. 25 4192-4207 (2018).

222. Puzyn, T. et al. Using nano-QSAR to predict the cytotoxicity of metal oxide nanoparticles. Nat. Nanotechnol. 6, 175-178 (2011).

223. Ahn, J. et al. Microfluidics in nanoparticle drug delivery; from synthesis to pre-clinical screening Adv. Drug Deliv. Rev. 128, 29-53 (2018).

224. He, Z., Ranganathan, N. \& Li, P. Evaluating nanomedicine with microfluidics. Nanotechnology 29 , 492001 (2018).

225. Carvalho, M. R. et al. Colorectal tumor-on-a-chip system: a 3D tool for precision onco-nanomedicine. Sci. Adv. 5, eaaw1317 (2019).

226. Dana, J., Agnus, V., Ouhmich, F. \& Gallix, B. Multimodality imaging and artificial intelligence for tumor characterization: current status and future perspective Semin. Nucl. Med. 50, 541-548 (2020).

227. Sun, R. et al. A radiomics approach to assess tumour-infiltrating $C D 8$ cells and response to anti-PD-1 or anti-PD-L1 immunotherapy: an imaging biomarker, retrospective multicohort study. Lancet Oncol. 19, 1180-1191 (2018)

228. Adir, O. et al. Integrating artificial intelligence and nanotechnology for precision cancer medicine. Adv. Mater. 32, 1901989 (2020).

229. Hong, M. K. H. et al. Tracking the origins and drivers of subclonal metastatic expansion in prostate cancer. Nat. Commun. 6, 6605 (2015).

230. Vignot, S., Besse, B., Andrē, F., Spano, J.-P. \& Soria, J.-C. Discrepancies between primary tumor and metastasis: a literature review on clinically established biomarkers. Crit. Rev. Oncol. Hematol. 84, 301-313 (2012).

231. Schroeder, A. et al. Treating metastatic cancer with nanotechnology. Nat. Rev. Cancer 12, 39-50 (2012)

232. Hu, Q. et al. Anticancer platelet-mimicking nanovehicles. Adv. Mater. 27, 7043-7050 (2015)

233. Liang, M. et al. H-ferritin-nanocaged doxorubicin nanoparticles specifically target and kill tumors with a single-dose injection. Proc. Natl Acad. Sci. USA 111 14900-14905 (2014).

234. Ahn, B. et al. Four-fold channel-nicked human ferritin nanocages for active drug loading and $\mathrm{pH}$-responsive drug release. Angew. Chem. Int. Ed. 57, 2909-2913 (2018)

235. Khatun, Z., Nurunnabi, M., Reeck, G. R., Cho, K. J. \& Lee, Y.-K. Oral delivery of taurocholic acid linked heparin-docetaxel conjugates for cancer therapy. J. Control. Rel. 170, 74-82 (2013).

236. Li, J. et al. Self-assembly of DNA nanohydrogels with controllable size and stimuli-responsive property for targeted gene regulation therapy. J. Am. Chem. Soc 137, 1412-1415 (2015)

237. Cutler, J. I., Auyeung, E. \& Mirkin, C. A. Spherical nucleic acids. J. Am. Chem. Soc. 134, 1376-1391 (2012).

238. Jiang, Q. et al. DNA origami as a carrier for circumvention of drug resistance. J. Am. Chem. Soc 134, 13396-13403 (2012).

239. Douglas, S. M. et al. Rapid prototyping of 3D DNA-origami shapes with caDNAno. Nucleic Acids Res. 37, 5001-5006 (2009).

240. Bindewald, E., Grunewald, C., Boyle, B., O'Connor, M $\&$ Shapiro, B. A. Computational strategies for the automated design of RNA nanoscale structures from building blocks using NanoTiler. J. Mol. Graph. Model. 27, 299-308 (2008)

241. Donnem, T. et al. Non-angiogenic tumours and their influence on cancer biology. Nat. Rev. Cancer 18 , 323-336 (2018).

242. Hida, K., Maishi, N., Torii, C. \& Hida, Y. Tumor angiogenesis - characteristics of tumor endothelial cells. Int. J. Clin. Oncol. 21, 206-212 (2016).

243. Joyce, J. A. \& Fearon, D. T. T cell exclusion, immune privilege, and the tumor microenvironment. Science 348, 74-80 (2015).

244. Gay, L. J. \& Felding-Habermann, B. Contribution of platelets to tumour metastasis. Nat. Rev. Cancer 11, 123-134 (2011).

245. Tozer, G. M., Kanthou, C. \& Baguley, B. C. Disrupting tumour blood vessels. Nat. Rev. Cancer 5, 423-435 (2005)

246. Nam, J. et al. Cancer nanomedicine for combination cancer immunotherapy. Nat. Rev. Mater. 4, 398-414 (2019).

247. Gajewski, T. F. Schreiber, H. \& Fu, Y-X. Innate and adaptive immune cells in the tumor microenvironment. Nat. Immunol. 14, 1014-1022 (2013).

248. Joshi, M. D., Unger, W. J., Storm, G., van Kooyk, Y. \& Mastrobattista, E. Targeting tumor antigens to dendritic cells using particulate carriers. J. Control. Rel. 161, 25-37 (2012).

249. Guillerey, C., Huntington, N. D. \& Smyth, M. J. Targeting natural killer cells in cancer immunotherapy. Nat. Immunol. 17, 1025-1036 (2016).

250. Ma, Q., Dieterich, L. C. \& Detmar, M. Multiple roles of lymphatic vessels in tumor progression. Curr Opin. Immunol. 53, 7-12 (2018).

251. Garnier, L., Gkountidi, A.-O. \& Hugues, S. Tumor-associated lymphatic vessel features and immunomodulatory functions. Front. Immunol. 10 , 720 (2019).

252. Laakkonen, P., Porkka, K., Hoffman, J. A. \& Ruoslahti, E. A tumor-homing peptide with a targeting specificity related to lymphatic vessels. Nat. Med. 8 $751-755$ (2002). 
253. Cheng, C. J., Tietjen, G. T., Saucier-Sawyer, J. K. \& Saltzman, W. M. A holistic approach to targeting disease with polymeric nanoparticles. Nat. Rev. Drug Discov. 14, 239 (2015)

254. Yang, Y. et al. Effect of size, surface charge, and hydrophobicity of poly(amidoamine) dendrimers on their skin penetration. Biomacromolecules 13 2154-2162 (2012)

255. De Matteis, V. Exposure to inorganic nanoparticles: routes of entry, immune response, biodistribution and in vitro/in vivo toxicity evaluation. Toxics 5, 29 (2017).

\section{Acknowledgements}

We sincerely acknowledge all researchers and their work in our field that we were unable to cite because of space limitations. This work was supported by the National Basic
Research Plan of China (2018YFA0208900), the National Key R\&D Program of China (2018YFE0205300), the Strategic Priority Research Program of Chinese Academy of Sciences (XDB36000000), the Key Research Program of Frontier Sciences CAS (ZDBS-LY-SLH039) and the K. C. Wong Education Foundation (GJTD-2018-03).

Author contributions

J.W. and Y.L. contributed equally to researching the data for the Review, discussing the content, and reviewing and editing the manuscript. G.N. conceived the structure and focus and was responsible for writing the Review. All authors approved the contents of the Review.

\section{Competing interests}

The authors declare no competing interests.
Publisher's note

Springer Nature remains neutral with regard to jurisdictional claims in published maps and institutional affiliations.

\section{RELATED LINKS}

caDNAno: https://cadnano.org/index.html

CanDo: https://cando-dna-origami.org/

ENERG-MD: http://bionano.physics.illinois.edu/origami-structure

Mfold: http://www.unafold.org/

NUPACK: http://www.nupack.org/

oxDNA: https://oxdna.org/

RNA designer: http://www.rnasoft.ca/cgi-bin/RNAsoft/

RNAdesigner/rnadesign.pl

Sfold: http://sfold.wadsworth.org/cgi-bin/index.pl

(c) Springer Nature Limited 2021 